\title{
DEFECTIVE COLOURING OF GRAPHS EXCLUDING A SUBGRAPH OR MINOR
}

\author{
PATRICE OSSONA DE MENDEZ, SANG-IL OUM, AND DAVID R. WOOD
}

\begin{abstract}
Archdeacon (1987) proved that graphs embeddable on a fixed surface can be 3-coloured so that each colour class induces a subgraph of bounded maximum degree. Edwards, Kang, Kim, Oum and Seymour (2015) proved that graphs with no $K_{t+1}$-minor can be $t$-coloured so that each colour class induces a subgraph of bounded maximum degree. We prove a common generalisation of these theorems with a weaker assumption about excluded subgraphs. This result leads to new defective colouring results for several graph classes, including graphs with linear crossing number, graphs with given thickness (with relevance to the earth-moon problem), graphs with given stack- or queue-number, linklessly or knotlessly embeddable graphs, graphs with given Colin de Verdière parameter, and graphs excluding a complete bipartite graph as a topological minor.
\end{abstract}

\section{INTRODUCTION}

A graph $G$ is $k$-colourable with defect $d$, or $d$-improper $k$-colourable, or simply $(k, d)$-colourable, if each vertex $v$ of $G$ can be assigned one of $k$ colours so that at most $d$ neighbours of $v$ are assigned the same colour as $v$. That is, each monochromatic subgraph has maximum degree at most $d$. Obviously the case $d=0$ corresponds to the usual notion of graph colouring. Cowen et al. 25] introduced the notion of defective graph colouring, and now many results for various graph classes are known. This paper presents $(k, d)$-colourability results for graph classes defined by an excluded subgraph, subdivision or minor. Our primary focus is on minimising the number of colours $k$ rather than the degree bound $d$. This viewpoint motivates the following definition. The defective chromatic number of a graph class $\mathcal{C}$ is the minimum integer $k$ (if such a $k$ exists) for which there exists an integer $d$ such that every graph in $\mathcal{C}$ is $(k, d)$-colourable.

Consider the following two examples: Archdeacon [3] proved that for every surface $\Sigma$, the defective chromatic number of graphs embeddable in $\Sigma$ equals 3 . And Edwards, Kang, Kim, Oum, and Seymour [31] proved that the class of graphs containing no $K_{t+1}$ minor has defective chromatic number $t$ (which is a weakening of Hadwiger's conjecture). This paper proves a general theorem that implies both these

Date: April 5, 2017.

Ossona de Mendez is supported by grant ERCCZ LL-1201 and by the European Associated Laboratory "Structures in Combinatorics" (LEA STRUCO), and partially supported by ANR project Stint under reference ANR-13-BS02-0007. Research of Wood is supported by the Australian Research Council. 


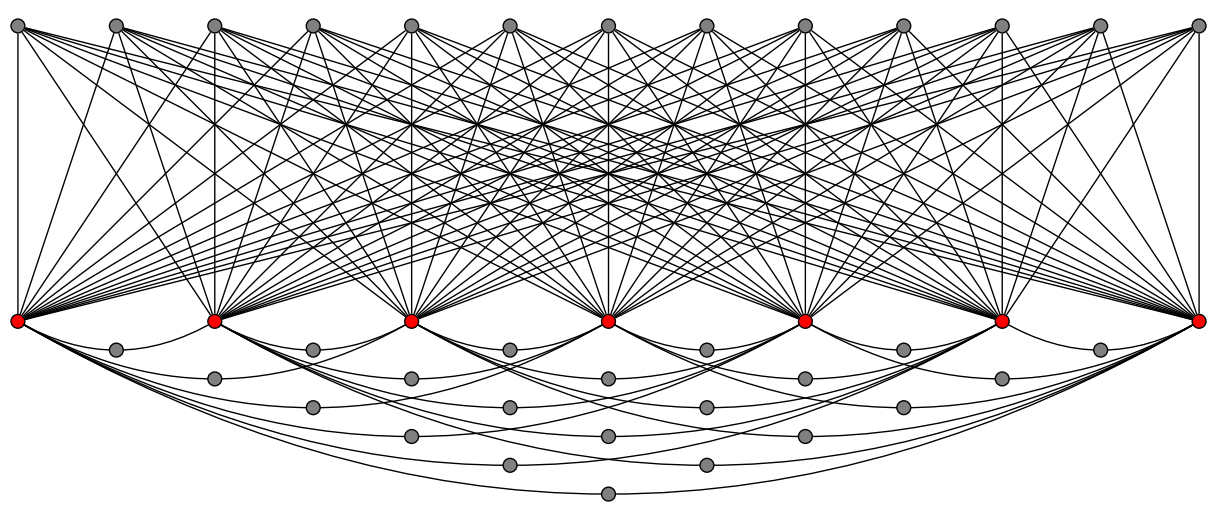

Figure 1. The graph $K_{7,13}^{*}$.

results as special cases. Indeed, our theorem only assumes an excluded subgraph, which enables it to be applied in more general settings.

For integers $s, t \geqslant 1$, let $K_{s, t}^{*}$ be the bipartite graph obtained from $K_{s, t}$ by adding $\left(\begin{array}{l}s \\ 2\end{array}\right)$ new vertices, each adjacent to a distinct pair of vertices in the colour class of $s$ vertices in $K_{s, t}$ (see Figure 1). Our main result shows that every graph excluding $K_{s, t}^{*}$ as a subgraph is $(s, d)$-colourable, where $d$ depends on $s, t$ and certain density parameters, which we now introduce.

For a graph $G$, the most natural density parameter to consider is the maximum average degree, denoted $\operatorname{mad}(G)$, which is the maximum of the average degrees of all subgraphs of $G$; that is,

$$
\operatorname{mad}(G):=\max _{H \subseteq G} \frac{2|E(H)|}{|V(H)|} .
$$

Closely related to maximum average degree is degeneracy. A graph $G$ is $k$-degenerate if every subgraph of $G$ has a vertex of degree at most $k$. Every graph is $\lfloor\operatorname{mad}(G)\rfloor$ degenerate. It follows that the chromatic number (and even the choice number) of a graph $G$ is at most $\lfloor\operatorname{mad}(G)+1\rfloor$. Bounds on defective colourings have also been obtained in terms of maximum average degree. In particular, Havet and Sereni [37] proved that every graph $G$ with $\operatorname{mad}(G)<k+\frac{k d}{k+d}$ is $(k, d)$-colourable, and that there exist non- $(k, d)$-colourable graphs whose maximum average degree tends to $2 k$ when $d$ goes to infinity, which shows the limit of the maximum average degree approach for defective colouring (see also [9, 12, 14, 19, 23, 28, 51]).

In addition to maximum average degree we consider the density of shallow topological minors (see [65] for more on this topic). A graph $H$ is a minor of a graph $G$ if a graph isomorphic to $H$ can be obtained from a subgraph of $G$ by contracting edges. A graph $H$ is a topological minor of a graph $G$ if a subdivision of $H$ is a subgraph of $G$. A $(\leqslant k)$-subdivision of a graph $G$ is a graph obtained from $G$ by subdividing each edge at most $k$ times, or equivalently replacing each edge by a path of length at most $k+1$. The exact 1-subdivision of $G$ is the graph obtained from $G$ by subdividing each edge exactly once. For a half integer $r$ (that is, a number $r$ 
such that $2 r$ is an integer), a graph $H$ is a depth-r topological minor of a graph $G$ if a $(\leqslant 2 r)$-subdivision of $H$ is a subgraph of $G$. For a graph $G$, let $G \widetilde{\nabla} r$ be the set of all depth- $r$ topological minors of $G$. The topological greatest reduced average density (or top-grad) with rank $r$ of a graph $G$ is defined as

$$
\widetilde{\nabla}_{r}(G):=\max _{H \in G \widetilde{\nabla} r} \frac{|E(H)|}{|V(H)|} .
$$

Note that $\widetilde{\nabla}_{1}(G) \geqslant \widetilde{\nabla}_{0}(G)=\frac{1}{2} \operatorname{mad}(G)$.

The following is our main result (see Theorem 2.3 for a more precise version).

Theorem 1.1. Every graph $G$ with no $K_{s, t}^{*}$ subgraph has an $(s, d)$-colouring, where $d$ depends on $s, t, \operatorname{mad}(G)$ and $\widetilde{\nabla}_{1 / 2}(G)$

We actually prove this result in the setting of defective list colourings, introduced by Eaton and Hull [30] and since studied by several authors [16 18, 37, 60, 80, 86, 88, 91 93. A $k$-list assignment of a graph $G$ is a function $L$ that assigns a set $L(v)$ of exactly $k$ colours to each vertex $v \in V(G)$. Then an $L$-colouring of $G$ is a function that assigns a colour in $L(v)$ to each vertex $v \in V(G)$. If an $L$-colouring has the property that every vertex $v$ has at most $d$ neighbours having the same colour as $v$, then we call it an $L$-colouring with defect $d$, or $d$-defective. A graph $G$ is $k$-choosable with defect $d$, or $d$-improper $k$-choosable, or simply $(k, d)$-choosable if for every $k$-list assignment $L$ of $G$, there exists a $d$-defective $L$-colouring of $G$. For example, the result of Havet and Sereni [37] mentioned above applies in the setting of $(k, d)$-choosability. The defective choice number of a graph class $\mathcal{C}$ is the minimum integer $k$ (if such a $k$ exists) for which there exists an integer $d$ such that every graph in $\mathcal{C}$ is $(k, d)$-choosable.

The paper is organised as follows. Section 2 presents the proof of our main result (a choosability version of Theorem 1.1). The subsequent sections present several applications of this main result. In particular, Section 3 gives results for graphs with no 4-cycle, and other graph classes defined by an excluded subgraph. Section 4 presents defective 3-colourability results for graphs drawn on surfaces, even allowing for a linear number of crossings, thus generalising the result of Archdeacon mentioned above. Section 5 gives bounds on the defective chromatic number and defective choice number of graphs with given thickness, and of graphs with given stack- or queue-number. One result here is relevant to the earth-moon problem, which asks for the chromatic number of graphs with thickness 2. While it is open whether such graphs are 11-colourable, we prove they are 11-colourable with defect 2. Section 6 studies the defective chromatic number of minor-closed classes. We determine the defective chromatic number and defective choice number of linklessly embeddable graphs, knotlessly embeddable graphs, and graphs with given Colin de Verdière parameter. We then prove a strengthening of the result of Edwards et al. 31] mentioned above. Finally, we formulate a conjecture about the defective chromatic number of $H$-minor-free graphs, and prove several special cases of it. 


\section{Main Proof}

An edge $e$ in a graph is $\ell$-light if both endpoints of $e$ have degree at most $\ell$. There is a large literature on light edges in graphs; see [8, 10, 15, 46, 48, 49] for example. Many of our results rely on the following sufficient condition for $(k, d)$-choosability. Its proof is essentially identical to the proof of a lemma by Lih et al. [60, Lemma 1]. Škrekovski [80] proved a similar result with $k=1$.

Lemma 2.1. For integers $\ell \geqslant k \geqslant 1$, if every subgraph $H$ of a graph $G$ has a vertex of degree at most $k$ or an $\ell$-light edge, then $G$ is $(k+1, \ell-k)$-choosable.

Proof. Let $L$ be a $(k+1)$-list assignment for $G$. We prove by induction on $|V(H)|+$ $|E(H)|$ that every subgraph $H$ of $G$ is $L$-colourable with defect $\ell-k$. The base case with $|V(H)|+|E(H)|=0$ is trivial. Consider a subgraph $H$ of $G$. If $H$ has a vertex $v$ of degree at most $k$, then by induction $H-v$ is $L$-colourable with defect $\ell-k$, and there is a colour in $L(v)$ used by no neighbour of $v$ which can be assigned to $v$. Now assume that $H$ has minimum degree at least $k+1$. By assumption, $H$ contains an $\ell$-light edge $x y$. By induction, $H-x y$ has an $L$-colouring $c$ with defect $\ell-k$. If $c(x) \neq c(y)$, then $c$ is also an $L$-colouring of $H$ with defect $\ell-k$. Now assume that $c(x)=c(y)$. We may further assume that $c$ is not an $L$-colouring of $H$ with defect $\ell-k$. Without loss of generality, $x$ has exactly $\ell-k+1$ neighbours (including $y$ ) coloured by $c(x)$. Since $\operatorname{deg}_{H}(x) \leqslant \ell$, there are at most $k-1$ neighbours not coloured by $c(x)$. Since $L(v)$ contains $k$ colours different from $c(x)$, there is a colour used by no neighbour of $x$ which can be assigned to $x$.

To state our main result, we use the following auxiliary function. For positive integers $s, t$ and positive reals $\delta$ and $\delta_{1}$, let

$$
N_{1}\left(s, t, \delta, \delta_{1}\right):= \begin{cases}(\delta-s)\left(\left(\begin{array}{l}
\left\lfloor\delta_{1}\right\rfloor \\
s-1
\end{array}\right)(t-1)+\frac{1}{2} \delta_{1}\right)+\delta & \text { if } s>2, \\
\frac{1}{2}(\delta-2) \delta_{1} t+\delta & \text { if } s=2, \\
t-1 & \text { if } s=1 .\end{cases}
$$

Lemma 2.2. For positive integers $s$, $t$, and positive reals $\delta$, $\delta_{1}$, let $\ell=\left\lfloor N_{1}\left(s, t, \delta, \delta_{1}\right)\right\rfloor$. If every subgraph of a graph $G$ has average degree at most $\delta$ and every graph whose exact 1-subdivision is a subgraph of $G$ has average degree at most $\delta_{1}$, then at least one of the following holds:

(i) $G$ contains a $K_{s, t}^{*}$ subgraph,

(ii) $G$ has a vertex of degree at most $s-1$,

(iii) $G$ has an $\ell$-light edge.

Proof. The case $s=1$ is simple: If (i) does not hold, then $\Delta(G) \leqslant t-1$, in which case either $G$ has no edges and (ii) holds, or $G$ has an edge and (iii) holds since $\ell=t-1$. Now assume that $s>1$.

Assume for contradiction that $G$ has no $K_{s, t}^{*}$ subgraph, that every vertex of $G$ has degree at least $s$ (thus $s \leqslant \delta$ ), and that $G$ contains no $\ell$-light edge. 
Let $A$ be the set of vertices in $G$ of degree at most $\ell$. Let $B:=V(G) \backslash A$. Let $a:=|A|$ and $b:=|B|$. Since $G$ has a vertex of degree at most $\delta$ and $\delta \leqslant \ell$, we deduce that $a>0$. Note that no two vertices in $A$ are adjacent.

Since the average degree of $G$ is at most $\delta$,

$$
(\ell+1) b+s a \leqslant 2|E(G)| \leqslant \delta(a+b) .
$$

That is,

$$
(\ell+1-\delta) b \leqslant(\delta-s) a .
$$

Let $G^{\prime}$ be a minor of $G$ obtained from $G-E(G[B])$ by greedily finding a vertex $w \in A$ having a pair of non-adjacent neighbours $x, y$ in $B$ and replacing $w$ by an edge joining $x$ and $y$ (by deleting all edges incident with $w$ except $x w, y w$ and contracting $x w)$, until no such vertex $w$ exists.

Let $A^{\prime}:=V\left(G^{\prime}\right) \backslash B$ and $a^{\prime}:=\left|A^{\prime}\right|$. Clearly the exact 1-subdivision of $G^{\prime}[B]$ is a subgraph of $G$. So every subgraph of $G^{\prime}[B]$ has average degree at most $\delta_{1}$. Since $G^{\prime}[B]$ contains at least $a-a^{\prime}$ edges,

$$
a-a^{\prime} \leqslant \frac{1}{2} \delta_{1} b
$$

A clique in a graph is a set of pairwise adjacent vertices. Let $M$ be the number of cliques of size $s$ in $G^{\prime}[B]$. Since $G^{\prime}[B]$ is $\left\lfloor\delta_{1}\right\rfloor$-degenerate,

$$
M \leqslant\left(\begin{array}{c}
\left\lfloor\delta_{1}\right\rfloor \\
s-1
\end{array}\right) b
$$

(see [65, p. 25] or [87]). If $s=2$, then the following better inequality holds:

$$
M \leqslant \frac{1}{2} \delta_{1} b .
$$

For each vertex $v \in A^{\prime}$, since $v$ was not contracted in the creation of $G^{\prime}$, the set of neighbours of $v$ in $B$ is a clique of size at least $s$. Thus if $a^{\prime}>M(t-1)$, then there are at least $t$ vertices in $A^{\prime}$ sharing at least $s$ common neighbours in $B$. These $t$ vertices and their $s$ common neighbours in $B$ with the vertices in $A-A^{\prime}$ form a $K_{s, t}^{*}$ subgraph of $G$, contradicting our assumption. Thus,

$$
a^{\prime} \leqslant M(t-1)
$$

By (1), (2) and (3),

$$
\ell+1 \leqslant(\delta-s)\left(\frac{M}{b}(t-1)+\frac{1}{2} \delta_{1}\right)+\delta,
$$

contradicting the definition of $\ell$.

Lemmas 2.1 and 2.2 imply our main result:

Theorem 2.3. For integers $s, t \geqslant 1$, every graph $G$ with no $K_{s, t}^{*}$ subgraph is $(s, d)$ choosable, where $d:=\left\lfloor N_{1}\left(s, t, \operatorname{mad}(G), 2 \widetilde{\nabla}_{1 / 2}(G)\right)\right\rfloor-s+1$. 
Proof. By definition, every subgraph of $G$ has average degree at most $\operatorname{mad}(G)$ and every graph whose exact 1-subdivision is a subgraph of $G$ has average degree at most $2 \widetilde{\nabla}_{1 / 2}(G)$. By Lemma 2.2 , every subgraph of $G$ has a vertex of degree at most $s-1$ or has an $\ell$-light edge, where $\ell:=\left\lfloor N_{1}\left(s, t, \operatorname{mad}(G), 2 \widetilde{\nabla}_{1 / 2}(G)\right)\right\rfloor$. By Lemma 2.1 with $k=s-1$, we have that $G$ is $(s, \ell-s+1)$-choosable.

The following recursive construction was used by Edwards et al. [31] to show that their theorem mentioned above is tight. We use this example repeatedly, so include the proof for completeness. If $s=2$, then let $G(s, N):=K_{1, N+1}$. If $s>2$, then let $G(s, N)$ be obtained from the disjoint union of $N+1$ copies of $G(s-1, N)$ by adding one new vertex $v$ adjacent to all other vertices. Note that Havet and Sereni [37] used a similar construction to prove their lower bound mentioned above.

Lemma 2.4 (Edwards et al. [31]). For integers $s \geqslant 2$ and $N \geqslant 1$, the graph $G=G(s, N)$ has no $K_{s, s}$ minor and no $(s-1, N)$-colouring.

Proof. We proceed by induction on $s$. In the base case, $G=K_{1, N+1}$, and every 1colouring has a colour class (the whole graph) inducing a subgraph with maximum degree larger than $N$. Thus $G$ is not $(1, N)$-colourable. Now assume that $s \geqslant 3$ and the claim holds for $s-1$. Let $v$ be the dominant vertex in $G$. Let $C_{1}, \ldots, C_{N+1}$ be the components of $G-v$, where each $C_{i}$ is isomorphic to $G(s-1, N)$.

If $G$ contains a $K_{s, s}$ minor, then some component of $G-v$ contains a $K_{s-1, s-1}$ minor, which contradicts our inductive assumption. Thus $G$ contains no $K_{s, s}$ minor.

Suppose that $c$ is an $(s-1)$-colouring of $G(s, N)$. We may assume that $c(v)=1$. If $C_{i}$ has a vertex of colour 1 for each $i \in\{1,2, \ldots, N+1\}$, then $v$ has more than $N$ neighbours of colour 1 , which is not possible. Thus some component $C_{i}$ has no vertex coloured 1 , and at most $s-2$ colours are used on $C_{i}$. This contradicts the assumption that $C_{i}$ has no $(s-2, N)$-colouring.

Of course, for integers $t \geqslant s \geqslant 2$, the graph $G(s, N)$ has no $K_{s, t}$ minor, no $K_{s, t}$ topological minor, and no $K_{s, t}^{*}$-minor. Thus Lemma 2.4 shows that the number of colours in Theorem 2.3 is best possible. In other words, Theorem 2.3 states that defective chromatic number and defective choice number of every class of graphs of bounded $\widetilde{\nabla}_{1 / 2}$ with no $K_{s, t}^{*}$ subgraph are at most $s$, and Lemma 2.4 shows that the number $s$ of colours cannot be decreased.

\section{ExCLUded SubGRAPHS}

This section presents several applications of our main result, in the setting of graph classes defined by an excluded subgraph. Since $K_{s, t}^{*}$ contains $K_{s, t}$ and a $(\leqslant 1)$-subdivision of $K_{s+1}$, Theorem 2.3 immediately implies:

- Every graph $G$ with no $K_{s, t}$ subgraph is $(s, d)$-choosable, where $d$ depends on $s, t, \operatorname{mad}(G)$ and $\widetilde{\nabla}_{1 / 2}(G)$.

- Every graph $G$ with no subgraph isomorphic to a $(\leqslant 1)$-subdivision of $K_{s+1}$ is $(s, d)$-choosable, where $d$ depends on $s, \operatorname{mad}(G)$ and $\widetilde{\nabla}_{1 / 2}(G)$. 
3.1. No 4-Cycles. Since $K_{2,1}^{*}$ is the 4-cycle, Theorem 2.3 with $s=2$ and $t=1$ implies the following.

Corollary 3.1. Every graph $G$ with no 4-cycle is $(2, d)$-choosable, where $d:=$ $\left\lfloor 2\left(\left(\widetilde{\nabla}_{0}(G)-1\right) \widetilde{\nabla}_{1 / 2}(G)+\widetilde{\nabla}_{0}(G)\right)-1\right\rfloor$.

Corollary 3.2. Every graph with no $K_{t}$ minor nor 4 -cycle subgraph is $\left(2, O\left(t^{2} \log t\right)\right)$ choosable.

For planar graphs, $\widetilde{\nabla}_{0} \leqslant \widetilde{\nabla}_{1 / 2} \leqslant 3$. Indeed $\widetilde{\nabla}_{0}<3$ for planar graphs with at least three vertices. Corollary 3.1 says that every planar graph with no 4-cycle is $(2,16)$ choosable. However, better degree bounds are known. Borodin et al. [11] proved that every planar graph with no cycle of length 4 has a vertex of degree at most 1 or a 7-light edge. By Lemma 2.1, planar graphs with no 4-cycle are (2,6)-choosable. Note that planar graphs with no 4-cycle are also known to be $(3,1)$-choosable 86 .

3.2. Edge Partitions. He et al. [38] proved the following theorem on partitioning a graph into edge-disjoint subgraphs.

Theorem 3.3 (He et al. [38, Theorem 3.1]). If every subgraph of a graph $G$ has a vertex of degree at most 1 or an $N$-light edge, then $G$ has an edge-partition into two subgraphs $T$ and $H$ such that $T$ is a forest and $H$ is a graph with maximum degree at most $N-1$.

This theorem and Lemma 2.2 imply the following.

Theorem 3.4. For an integer $t \geqslant 2$, every graph $G$ with no $K_{2, t}$ subgraph has an edge-partition into two subgraphs $T$ and $H$ such that $T$ is a forest and $H$ has maximum degree at most $\left\lfloor\left(\widetilde{\nabla}_{0}(G)-1\right) \widetilde{\nabla}_{1 / 2}(G)(t-1)+2 \widetilde{\nabla}_{0}(G)\right\rfloor-1$.

3.3. Nowhere Dense Classes. A class $\mathcal{C}$ of graphs is nowhere dense [63] if, for every integer $k$ there is some $n$ such that no $(\leqslant k)$-subdivision of $K_{n}$ is a subgraph of a graph in $\mathcal{C}$. Nowhere dense classes are also characterised [64] by the property that for every integer $r$ there exists a function $f_{r}: \mathbb{N} \rightarrow[0,1]$ with $\lim _{n \rightarrow \infty} f_{r}(n)=0$ such that every graph $G$ of order $n$ in the class has $\widetilde{\nabla}_{r}(G) \leqslant n^{f_{r}(n)}$. In other words, for each integer $r$ every graph $G$ in the class has $\widetilde{\nabla}_{r}(G)=|V(G)|^{o(1)}$.

For nowhere dense classes, there is no hope to find an improper colouring with a bounded number of colours, since the chromatic number of a nowhere dense class is typically unbounded (as witnessed by the class of graphs $G$ such that $\Delta(G) \leqslant$ $\operatorname{girth}(G))$. However, by the above characterisation, Theorem 2.3 implies there is a partition of the vertex set into a bounded number of parts, each with 'small' maximum degree.

Corollary 3.5. Let $\mathcal{C}$ be a nowhere dense class. Then there exist $c \in \mathbb{N}$ and $a$ function $f: \mathbb{N} \rightarrow[0,1]$ with $\lim _{n \rightarrow \infty} f(n)=0$ such that every $n$-vertex graph in $\mathcal{C}$ is $\left(c, n^{f(n)}\right)$-choosable. 


\section{3-Colouring Graphs on Surfaces}

This section considers defective colourings of graphs drawn on a surface, possibly with crossings. First consider the case of no crossings. For example, Cowen et al. [25] proved that every planar graph is $(3,2)$-colourable, improved to $(3,2)$-choosable by Eaton and Hull [30]. Since $G(3, N)$ is planar, by Lemma 2.4 the class of planar graphs has defective chromatic-number and defective choice number equal to 3 . More generally, Archdeacon [3] proved the conjecture of Cowen et al. 25] that for every fixed surface $\Sigma$, the class of graphs embeddable in $\Sigma$ has defective chromaticnumber 3. Woodall [89] proved that such graphs have defective choice number 3. It follows from Euler's formula that $K_{3, t}$ is not embeddable on $\Sigma$ for some constant $t$ (see Lemma 4.3), and that graphs embeddable in $\Sigma$ have bounded average degree and $\widetilde{\nabla}_{1 / 2}$. Thus Theorem 2.3 implies Woodall's result. The lower bound follows from Lemma 2.4 since $G(3, N)$ is planar.

Theorem 4.1 ([3, 89]). For every surface $\Sigma$, the class of graphs embeddable in $\Sigma$ has defective chromatic-number 3 and defective choice number 3.

While our main goal is to bound the number of colours in a defective colouring, we now estimate the degree bound using our method for a graph embeddable in a surface $\Sigma$ of Euler genus $g$. The Euler genus of an orientable surface with $h$ handles is $2 h$. The Euler genus of a non-orientable surface with $c$ cross-caps is $c$. The Euler genus of a graph $G$ is the minimum Euler genus of a surface in which $G$ embeds. For $g \geqslant 0$, define

$$
d_{g}:=\max \left\{3, \frac{1}{4}(5+\sqrt{24 g+1})\right\} .
$$

The next two lemmas are well known. We include their proofs for completeness.

Lemma 4.2. Every n-vertex graph $G$ embeddable in a surface of Euler genus $g$ has at most $d_{g} n$ edges.

Proof. Suppose that $|E(G)|>d n$, where $d:=d_{g}$. We may assume that $n \geqslant 3$. By Euler's Formula, $d n<|E(G)| \leqslant 3(n+g-2)$, implying $(d-3) n<3 g-6$. Since $d n<|E(G)| \leqslant\left(\begin{array}{l}n \\ 2\end{array}\right)$ we have $n>2 d+1$. Since $d \geqslant 3$,

$$
3 g-6>(d-3) n \geqslant(d-3)(2 d+1)=2 d^{2}-5 d-3 .
$$

Thus $2 d^{2}-5 d+(3-3 g)<0$. By the quadratic formula, $d<\frac{1}{4}(5+\sqrt{1+24 g})$, which is a contradiction. Hence $|E(G)| \leqslant d n$.

Lemma 4.3 (Ringel [73]). For every surface $\Sigma$ of Euler genus g, the complete bipartite graph $K_{3,2 g+3}$ does not embed in $\Sigma$.

Proof. By Euler's formula, every triangle-free graph with $n \geqslant 3$ vertices that embeds in $\Sigma$ has at most $2(n+g-2)$ edges. The result follows.

Lemmas 4.2 and 4.3 and Theorem 2.3 imply that graphs embeddable in $\Sigma$ are $\left(3, O\left(g^{5 / 2}\right)\right)$-choosable. This degree bound is weaker than the bound of $\max \left\{15, \frac{1}{2}(3 g-\right.$ 
8)\} obtained by Archdeacon. However, our bound is easily improved. Results by Jendrol' and Tuhársky [47] and Ivančo [43] show that every graph with Euler genus $g$ has a $(2 g+8)$-light edge. Then Lemma 2.1 directly implies that every graph with Euler genus $g$ is $(3,2 g+6)$-choosable. Still this bound is weaker than the subsequent improvements to Archdeacon's result of $(3, \max \{12,6+\sqrt{6 g}\})$-colourability by Cowen et al. [24] and to $(3, \max \{9,2+\sqrt{4 g+6}\})$-choosability by Woodall [89]; also see [18].

4.1. Linear Crossing Number. We now generalise Theorem 4.1 to the setting of graphs with linear crossing number. For an integer $g \geqslant 0$ and real number $k \geqslant 0$, say a graph $G$ is $k$-close to Euler genus $g$ (resp. $k$-close to planar) if every subgraph $H$ of $G$ has a drawing on a surface of Euler genus $g$ (resp. on the plane) with at most $k|E(H)|$ crossings. This says that the average number of crossings per edge is at most $2 k$ (for every subgraph). Of course, a graph is planar if and only if it is 0-close to planar, and a graph has Euler genus at most $g$ if and only if it is 0 -close to Euler genus $g$. Graphs that can be drawn in the plane with at most $k$ crossings per edge, so called $k$-planar graphs, are examples of graphs $\left(\frac{k}{2}\right)$-close to planar. Pach and Tóth [68] proved that $k$-planar graphs have average degree $O(\sqrt{k})$. It follows that $k$-planar graphs are $O(\sqrt{k})$-colourable, which is best possible since $K_{n}$ is $O\left(n^{2}\right)$-planar. For defective colourings, three colours suffice even in the more general setting of graphs $k$-close to Euler genus $g$.

Theorem 4.4. For all integers $g, k \geqslant 0$ the class of graphs $k$-close to Euler genus $g$ has defective chromatic number and defective choice number equal to 3. In particular, every graph $k$-close to Euler genus $g$ is $\left(3, O\left((k+1)^{5 / 2}(g+1)^{7 / 2}\right)\right)$-choosable.

We prove this theorem by a series of lemmas, starting with a straightforward extension of the standard probabilistic proof of the crossing lemma. Note that Shahrokhi et al. [79] obtained a better bound for a restricted range of values for $m$ relative to $n$.

Lemma 4.5. Every drawing of a graph with $n$ vertices and $m \geqslant 2 d_{g} n$ edges on a surface $\Sigma$ of Euler genus $g$ has at least $m^{3} /\left(8\left(d_{g} n\right)^{2}\right)$ crossings.

Proof. By Lemma 4.2, every $n$-vertex graph that embeds in $\Sigma$ has at most $d_{g} n$ edges. Thus every drawing of an $n$-vertex $m$-edge graph on $\Sigma$ has at least $m-d_{g} n$ crossings.

Given a graph $G$ with $n$ vertices and $m \geqslant 2 d_{g} n$ edges and a crossing-minimal drawing of $G$ on $\Sigma$, choose each vertex of $G$ independently and randomly with probability $p:=2 d_{g} n / m$. Note that $p \leqslant 1$. Let $G^{\prime}$ be the induced subgraph obtained. The expected number of vertices in $G^{\prime}$ is $p n$, the expected number of edges in $G^{\prime}$ is $p^{2} m$, and the expected number of crossings in the induced drawing of $G^{\prime}$ is $p^{4} c$, where $c$ is the number of crossings in the drawing of $G$. By linearity of expectation and the above naive bound, $p^{4} c \geqslant p^{2} m-d_{g} p n$. Thus $c \geqslant\left(p m-d_{g} n\right) / p^{3}=d_{g} n / p^{3}=$ $m^{3} /\left(8\left(d_{g} n\right)^{2}\right)$.

This lemma leads to the following bound on the number of edges. 
Lemma 4.6. If an n-vertex $m$-edge graph $G$ has a drawing on a surface of Euler genus $g$ with at most $k m$ crossings, then

$$
m \leqslant \sqrt{8 k+4} d_{g} n .
$$

Proof. If $m<2 d_{g} n$ then $m<\sqrt{8 k+4} d_{g} n$, and we are done. Otherwise, $m \geqslant 2 d_{g} n$, and Lemma 4.5 is applicable. Thus every drawing of $G$ on a surface of Euler genus $g$ has at least $m^{3} /\left(8\left(d_{g} n\right)^{2}\right)$ crossings. Hence $m^{3} /\left(8\left(d_{g} n\right)^{2}\right) \leqslant k m$, implying $m \leqslant$ $\sqrt{8 k} d_{g} n$.

To apply Theorem 2.3 we bound the size of $K_{3, t}$ subgraphs.

Lemma 4.7. Every drawing of $K_{3, t}$ in a surface of Euler genus $g$ has at least

$$
\frac{t(t-1)}{(2 g+3)(2 g+2)}
$$

crossings.

Proof. By Lemma $4.3, K_{3,2 g+3}$ does not embed (crossing-free) in a surface of Euler genus $g$. Consider a drawing of $K_{3, t}$ in a surface of Euler genus $g$. There are $\left(\begin{array}{c}t \\ 2 g+3\end{array}\right)$ copies of $K_{3,2 g+3}$ in $K_{3, t}$. Each such copy has a crossing. Each crossing is in at most $\left(\begin{array}{c}t-2 \\ 2 g+1\end{array}\right)$ copies of $K_{3,2 g+3}$. Thus the number of crossings is at least

$$
\left(\begin{array}{c}
t \\
2 g+3
\end{array}\right) /\left(\begin{array}{c}
t-2 \\
2 g+1
\end{array}\right)=\frac{t(t-1)}{(2 g+3)(2 g+2)} .
$$

Lemma 4.8. If a graph $G$ is $k$-close to Euler genus $g$ and contains $K_{3, t}$ as a subgraph, then

$$
t \leqslant 3 k(2 g+3)(2 g+2)+1 .
$$

Proof. Suppose that $G$ contains $K_{3, t}$ as a subgraph. Since $G$ is $k$-close to Euler genus $g$, so is $K_{3, t}$. Thus $K_{3, t}$ has a drawing in a surface of Euler genus $g$ where the number of crossings is at most $3 k t$. By Lemma 4.7.

$$
\frac{t(t-1)}{(2 g+3)(2 g+2)} \leqslant 3 k t .
$$

The result follows.

We now prove the main result of this section.

Proof of Theorem 4.4. Say $G$ is a graph $k$-close to Euler genus $g$. By Lemma 4.8 , $G$ contains no $K_{3, t}$ with $t=3 k(2 g+3)(2 g+2)+2$. By Lemma 4.6 , $\operatorname{mad}(G) \leqslant$ $2 \sqrt{8 k+4} d_{g}$. We now bound $\widetilde{\nabla}_{1 / 2}(G)$. Consider a subgraph $H$ of $G$ that is a $(\leqslant 1)-$ subdivision of a graph $X$. Since $G$ is $k$-close to Euler genus $g$, so is $H$. Thus $H$ has a drawing on a surface of Euler genus $g$ with at most $k|E(H)|$ crossings. Remove each division vertex and replace its two incident edges by one edge. We obtain a drawing of $X$ with the same number of crossings as the drawing of $H$. Now $|E(H)| \leqslant 2|E(X)|$. Thus $X$ has a drawing on a surface of Euler genus $g$ with 
at most $2 k|E(X)|$ crossings. By Lemma $4.6,|E(X)| \leqslant \sqrt{16 k+4} d_{g}|V(X)|$. Hence $\widetilde{\nabla}_{1 / 2}(G) \leqslant \sqrt{16 k+4} d_{g}$. By Theorem 2.3. $G$ is $(3, d)$-choosable, where

$$
\begin{aligned}
d & =\left\lfloor N_{1}\left(3,3 k(2 g+3)(2 g+2)+2,2 \sqrt{8 k+4} d_{g}, 2 \sqrt{16 k+4} d_{g}\right)\right\rfloor-2 \\
& \leqslant O\left((k+1)^{5 / 2}(g+1)^{7 / 2}\right) .
\end{aligned}
$$

\section{Thickness Parameters}

This section studies defective colourings of graphs with given thickness or other related parameters. Yancey [90] first proposed studying defective colourings of graphs with given thickness.

5.1. Light Edge Lemma. Our starting point is the following sufficient condition for a graph to have a light edge. The proof uses a technique by Bose et al. [15], which we present in a general form.

Lemma 5.1. Let $G$ be a graph with $n$ vertices, at most an $+b$ edges, and minimum degree $\delta$, such that every spanning bipartite subgraph has at most $a^{\prime} n+b^{\prime}$ edges, for some $a, a^{\prime} \in \mathbb{R}^{+}$and $b, b^{\prime} \in \mathbb{R}$ and $\delta \in \mathbb{Z}^{+}$satisfying:

$$
\begin{aligned}
& 2 a \geqslant \delta>a^{\prime}, \\
& \left(\delta-a^{\prime}\right) \ell>\left(2 a-a^{\prime}\right) \delta, \text { and } \\
& \left(\delta-a^{\prime}\right) \ell^{2}-\left(\left(2 a-a^{\prime}\right) \delta+b^{\prime}-\delta+a^{\prime}\right) \ell-\left(2 a-a^{\prime}+2 b-b^{\prime}\right) \delta>0 .
\end{aligned}
$$

Then $G$ has an $(\ell-1)$-light edge.

Proof. Let $X$ be the set of vertices with degree at most $\ell-1$. Since vertices in $X$ have degree at least $\delta$ and vertices not in $X$ have degree at least $\ell$,

$$
\delta|X|+(n-|X|) \ell \leqslant \sum_{v \in V(G)} \operatorname{deg}(v)=2|E(G)| \leqslant 2(a n+b) .
$$

Thus

$$
(\ell-2 a) n-2 b \leqslant(\ell-\delta)|X| .
$$

Suppose on the contrary that $X$ is an independent set in $G$. Let $G^{\prime}$ be the spanning bipartite subgraph of $G$ consisting of all edges between $X$ and $V(G) \backslash X$. Since each of the at least $\delta$ edges incident with each vertex in $X$ are in $G^{\prime}$,

$$
\delta|X| \leqslant\left|E\left(G^{\prime}\right)\right| \leqslant a^{\prime} n+b^{\prime} .
$$

Since $\ell>\frac{2 a-a^{\prime}}{\delta-a^{\prime}} \delta>\delta$ (hence $\ell-\delta>0$ ) and $\delta \geqslant 0$,

$$
\begin{array}{rlrl} 
& & \delta(\ell-2 a) n-2 b \delta \leqslant \delta(\ell-\delta)|X| & \leqslant(\ell-\delta)\left(a^{\prime} n+b^{\prime}\right) \\
\Rightarrow & & \left(\delta(\ell-2 a)-a^{\prime}(\ell-\delta)\right) n \leqslant(\ell-\delta) b^{\prime}+2 b \delta \\
\Rightarrow & & \left(\left(\delta-a^{\prime}\right) \ell-\left(2 a-a^{\prime}\right) \delta\right) n \leqslant b^{\prime} \ell+\left(2 b-b^{\prime}\right) \delta .
\end{array}
$$

If $n \leqslant \ell$ then every edge is $(\ell-1)$-light. Now assume that $n \geqslant \ell+1$. Since $\left(\delta-a^{\prime}\right) \ell-\left(2 a-a^{\prime}\right) \delta>0$,

$$
\left(\left(\delta-a^{\prime}\right) \ell-\left(2 a-a^{\prime}\right) \delta\right)(\ell+1) \leqslant b^{\prime} \ell+\left(2 b-b^{\prime}\right) \delta .
$$


Thus

$$
\left(\delta-a^{\prime}\right) \ell^{2}+\left(\delta-a^{\prime}-\left(2 a-a^{\prime}\right) \delta-b^{\prime}\right) \ell \leqslant\left(2 a-a^{\prime}+2 b-b^{\prime}\right) \delta,
$$

which is a contradiction. Thus $X$ is not an independent set. Hence $G$ contains an $(\ell-1)$-light edge.

Remark. To verify (6), the following approximation can be useful: If $\alpha, \beta, \gamma$ are strictly positive reals, then the larger root of $\alpha x^{2}-\beta x-\gamma=0$ is at most

$$
\frac{\beta+\sqrt{\beta\left(\beta+\frac{4 \alpha \gamma}{\beta}\right)}}{2 \alpha} \leqslant \frac{\beta+\frac{1}{2}\left(\beta+\left(\beta+\frac{4 \alpha \gamma}{\beta}\right)\right)}{2 \alpha}=\frac{\beta}{\alpha}+\frac{\gamma}{\beta} .
$$

Lemma 2.1 with $k=\delta-1$ and Lemma 5.1 imply the following sufficient condition for defective choosability. With $\delta:=\left\lfloor a^{\prime}\right\rfloor+1$, which is the minimum possible value for $\delta$, the number of colours only depends on the coefficient of $|V(H)|$ in the bound on the number of edges in a bipartite subgraph $H$.

Lemma 5.2. Fix constants $a, a^{\prime} \in \mathbb{R}^{+}$and $b, b^{\prime} \in \mathbb{R}$ and $\ell, \delta \in \mathbb{Z}^{+}$satisfying (4), (5) and (6). Let $G$ be a graph such that every subgraph $H$ of $G$ with minimum degree at least $\delta$ satisfies the following conditions:

(i) $H$ has at most $a|V(H)|+b$ edges.

(ii) Every spanning bipartite subgraph of $H$ has at most $a^{\prime}|V(H)|+b^{\prime}$ edges.

Then $G$ is $(\delta, \ell-\delta)$-choosable. In particular, $G$ is $\left(\left\lfloor a^{\prime}\right\rfloor+1, \ell-1-\left\lfloor a^{\prime}\right\rfloor\right)$-choosable.

Lemma 5.1 with $a=3$ and $b=3(g-2)$ and $a^{\prime}=2$ and $b^{\prime}=2(g-2)$ and $\ell=2 g+13$ implies that every graph $G$ with minimum degree at least 3 and Euler genus $g$ has a $(2 g+12)$-light edge. Note that this bound is within +10 of being tight since $K_{3,2 g+2}$ has minimum degree 3, embeds in a surface of Euler genus $g$, and every edge has an endpoint of degree $2 g+2$. More precise results, which are typically proved by discharging with respect to an embedding, are known [8, 43, 47]. Lemma 5.2 then implies that every graph with Euler genus $g$ is $(3,2 g+10)$-choosable. As mentioned earlier, this result with a better degree bound was proved by Woodall [89]; also see [18]. The utility of Lemma 5.2 is that it is immediately applicable in more general settings, as we now show.

5.2. Thickness. The thickness of a graph $G$ is the minimum integer $k$ such that $G$ is the union of $k$ planar subgraphs; see [62] for a survey on thickness. A minimumdegree-greedy algorithm properly $6 k$-colours a graph with thickness $k$, and it is an open problem to improve this bound for $k \geqslant 2$. The result of Havet and Sereni [37] implies that graphs with thickness $k$, which have maximum average degree less than $6 k$, are $\left(3 k+1, O\left(k^{2}\right)\right)$-choosable, but gives no result with at most $3 k$ colours. We show below that graphs with thickness $k$ are $\left(2 k+1, O\left(k^{2}\right)\right)$-choosable, and that no result with at most $2 k$ colours is possible. That is, both the defective chromatic number and defective choice number of the class of graphs of thickness at most $k$ equal $2 k+1$. In fact, the proof works in the following more general setting. For an integer $g \geqslant 0$, the $g$-thickness of a graph $G$ is the minimum integer $k$ such that 
$G$ is the union of $k$ subgraphs each with Euler genus at most $g$. This definition was implicitly introduced by Jackson and Ringel [44]. By Euler's Formula, every graph with $n \geqslant 3$ vertices and $g$-thickness $k$ has at most $3 k(n+g-2)$ edges, and every spanning bipartite subgraph has at most $2 k(n+g-2)$ edges. Lemma 5.1 with $\ell=2 k g+8 k^{2}+4 k+1$ (using (7) to verify (6)) implies:

Lemma 5.3. Every graph with minimum degree at least $2 k+1$ and $g$-thickness at most $k$ has a $\left(2 k g+8 k^{2}+4 k\right)$-light edge.

We now determine the defective chromatic number and defective choice number of graphs with given $g$-thickness.

Theorem 5.4. For integers $g \geqslant 0$ and $k \geqslant 1$, the class of graphs with $g$-thickness at most $k$ has defective chromatic number and defective choice number equal to $2 k+1$. In particular, every graph with $g$-thickness at most $k$ is $\left(2 k+1,2 k g+8 k^{2}+2 k\right)$ choosable.

Proof. Lemmas 5.2 and 5.3 imply the upper bound. As usual, the lower bound is provided by $G(2 k+1, N)$. We now prove that $G=G(2 k+1, N)$ has $g$-thickness at most $k$ by induction on $k$ (with $g$ fixed). Note that $G(3, N)$ is planar, and thus has $g$-thickness 1. Let $r$ be the vertex of $G$ such that $G-r$ is the disjoint union of $N+1$ copies of $G(2 k, N)$. For $i \in[N+1]$, let $v_{i}$ be the vertex of the $i$-th component $C_{i}$ of $G-r$ such that $C_{i}-v_{i}$ is the disjoint union of $N+1$ copies of $G(2 k-1, N)$. Let $H:=G-\left\{r, v_{1}, v_{2}, \ldots, v_{N+1}\right\}$. Observe that each component of $H$ is isomorphic to $G(2 k-1, N)$ and by induction, $H$ has $g$-thickness at most $k-1$. Since $G-E(H)$ consists of $N+1$ copies of $K_{2, N^{\prime}}$ pasted on $r$ for some $N^{\prime}, G-E(H)$ is planar and thus has $g$-thickness 1 . Hence $G$ has $g$-thickness at most $k$. By Lemma 2.4 , $G(2 k+1, N)$ has no $(2 k, N)$-colouring. Therefore the class of graphs with $g$-thickness at most $k$ has defective chromatic number and defective choice number at least $2 k+1$.

The case $g=0$ and $k=2$ relates to the famous earth-moon problem [2, 34, 42, 44, 72, which asks for the maximum chromatic number of graphs with thickness 2 . The answer is in $\{9,10,11,12\}$. The result of Havet and Sereni [37] mentioned in Section1 implies that graphs with thickness 2 are $(7,18)$-choosable, $(8,9)$-choosable, $(9,5)$ choosable, (10,3)-choosable, and $(11,2)$-choosable because their maximum average degree is less than 12. But their result gives no bound with at most 6 colours. Theorem 5.4 says that the class of graphs with thickness 2 has defective chromatic number and defective choice number equal to 5 . In particular, Lemma 5.2 implies that graphs with thickness 2 are $(5,36)$-choosable, $(6,19)$-choosable, $(7,12)$-choosable, $(8,9)$-choosable, $(9,6)$-choosable, $(10,4)$-choosable, and $(11,2)$-choosable. This final result, which is also implied by the result of Havet and Sereni [37], is very close to the conjecture that graphs with thickness 2 are 11-colourable. Improving these degree bounds provides an approach for attacking the earth-moon problem. 
5.3. Stack Layouts. A $k$-stack layout of a graph $G$ consists of a linear ordering $v_{1}, \ldots, v_{n}$ of $V(G)$ and a partition $E_{1}, \ldots, E_{k}$ of $E(G)$ such that no two edges in $E_{i}$ cross with respect to $v_{1}, \ldots, v_{n}$ for each $i \in[1, k]$. Here edges $v_{a} v_{b}$ and $v_{c} v_{d}$ cross if $a<c<b<d$. A graph is a $k$-stack graph if it has a $k$-stack layout. The stack-number of a graph $G$ is the minimum integer $k$ for which $G$ is a $k$-stack graph. Stack layouts are also called book embeddings, and stack-number is also called bookthickness, fixed outer-thickness and page-number. The maximum chromatic number of $k$-stack graphs is in $\{2 k, 2 k+1,2 k+2\}$; see [29]. For defective colourings, $k+1$ colours suffice.

Theorem 5.5. The class of $k$-stack graphs has defective chromatic number and defective choice number equal to $k+1$. In particular, every $k$-stack graph is $(k+$ $\left.1,2^{O(k \log k)}\right)$-choosable.

Proof. The lower bound follows from Lemma 2.4 since an easy inductive argument shows that $G(k+1, N)$ is a $k$-stack graph for all $N$. For the upper bound, $K_{k+1, k(k+1)+1}$ is not a $k$-stack graph [5]; see also [26]. Every $k$-stack graph $G$ has average degree less than $2 k+2$ (see [5, 29, 50]) and $\widetilde{\nabla}_{1 / 2}(G) \leqslant 20 k^{2}$ (see [66]). The result follows from Theorem 2.3 with $s=k+1$ and $t=k(k+1)+1$, where $\left\lfloor N_{1}\left(k+1, k(k+1)+1,2 k+2,40 k^{2}\right)\right\rfloor-k \leqslant 2^{O(k \log k)}$.

5.4. Queue Layouts. A $k$-queue layout of a graph $G$ consists of a linear ordering $v_{1}, \ldots, v_{n}$ of $V(G)$ and a partition $E_{1}, \ldots, E_{k}$ of $E(G)$ such that no two edges in $E_{i}$ are nested with respect to $v_{1}, \ldots, v_{n}$ for each $i \in[1, k]$. Here edges $v_{a} v_{b}$ and $v_{c} v_{d}$ are nested if $a<c<d<b$. The queue-number of a graph $G$ is the minimum integer $k$ for which $G$ has a $k$-queue layout. A graph is a $k$-queue graph if it has a $k$-queue layout. Dujmović and Wood [29] state that determining the maximum chromatic number of $k$-queue graphs is an open problem, and showed lower and upper bounds of $2 k+1$ and $4 k$. We provide the following partial answer to this question.

Theorem 5.6. Every $k$-queue graph is $\left(2 k+1,2^{O(k \log k)}\right)$-choosable.

Proof. Heath and Rosenberg [40] proved that $K_{2 k+1,2 k+1}$ is not a $k$-queue graph. Every $k$-queue graph $G$ has $\operatorname{mad}(G)<4 k$ (see [29, 40, 69]) and $\widetilde{\nabla}_{1 / 2}(G)<(2 k+2)^{2}$ (see [66]). The result then follows from Theorem 2.3 with $s=2 k+1$ and $t=2 k+1$, where $\left\lfloor N_{1}\left(2 k+1,2 k+1,4 k, 2(2 k+2)^{2}\right)\right\rfloor-2 k \leqslant 2^{O(k \log k)}$.

Since $G(k+1, n)$ has a $k$-queue layout, the defective chromatic number of the class of $k$-queue graphs is at least $k+1$ and at most $2 k+1$ by Lemma 2.4 and Theorem 5.6 . It remains an open problem to determine its defective chromatic number.

5.5. Posets. Consider the problem of partitioning the domain $X$ of a given poset $P=(X, \preceq)$ into $X_{1}, \ldots, X_{k}$ so that each $\left(X_{i}, \preceq\right)$ has small poset dimension. The Hasse diagram $H(P)$ of $P$ is the graph whose vertices are the elements of $P$ and whose edges correspond to the cover relation of $P$. Here $x$ covers $y$ in $P$ if $y \neq x$, $y \preceq x$ and there is no element $z$ of $P$ such that $z \neq y, z \neq x$, and $y \preceq z \preceq x$. A 
linear extension of $P=(X, \preceq)$ is a total order $\leq$ on $X$ such that $x \preceq y$ implies $x \leq y$ for every $x, y \in X$. The jump number of $P$ is the minimum number of consecutive elements of a linear extension of $P$ that are not comparable in $P$, where the minimum is taken over all possible linear extensions of $P$.

Theorem 5.7. For every integer $k$ there is an integer $d$ such that the domain of any poset with jump number at most $k$ can be coloured with $2 k+3$ colours, such that each colour induces a poset with dimension at most $d$.

Proof. Heath and Pemmaraju [39] showed that the queue-number of the Hasse diagram of a poset $P$ is at most one more than the jump number of $P$, and Füredi and Kahn [33] proved that if the Hasse diagram of a poset has maximum degree $\Delta$, then its dimension is at most $50 \Delta(\log \Delta)^{2}$. The result then follows from Theorem 5.6 .

\section{Minor-Closed Classes}

This section shows that for many minor-closed classes, Theorem 2.3 determines the defective chromatic number and defective choice number. For example, every outerplanar graph has average degree less than 4 and contains no $K_{2,3}$ subgraph. Thus Theorem 2.3 implies that every outerplanar graph is $(2,14)$-choosable. A better degree bound was obtained by Cowen et al. [25], who proved that outerplanar graphs are $(2,2)$-colourable. Since $G(1, N)$ is outerplanar, by Lemma 2.4 the defective chromatic number and defective choice number of the class of outerplanar graphs equal 2. As shown in Section 4, the defective chromatic number and defective choice number of the class of graphs embeddable in any fixed surface equal 3 . We now consider some other minor-closed classes.

6.1. Linklessly and Knotlessly Embeddable Graphs. A graph is linklessly embeddable if it has an embedding in $\mathbb{R}^{3}$ with no two topologically linked cycles [75, 77]. Linklessly embeddable graphs form a minor-closed class whose minimal excluded minors are the so-called Petersen family [76], which includes $K_{6}, K_{4,4}$ minus an edge, and the Petersen graph. Since linklessly embeddable graphs exclude $K_{6}$ minors, they are 5-colourable [74] and 8-choosable [4]. It is open whether $K_{6}$-minor-free graphs or linklessly embeddable graphs are 6-choosable. A graph is apex if deleting at most one vertex makes it planar. Every apex graph is linklessly embeddable [75]. Since $G(3, N)$ is planar, $G(4, N)$ is apex, and thus linklessly embeddable. By Lemma 2.4 . the class of linklessly embeddable graphs has defective chromatic number at least 4 . Mader's theorem [61] for $K_{6}$-minor-free graphs implies that linklessly embeddable graphs have average degree less than 8 and minimum degree at most 7 . Since linklessly embeddable graphs exclude $K_{4,4}$ minors, Theorem 2.3 implies the following result.

Theorem 6.1. The class of linklessly embeddable graphs has defective chromatic number and defective choice number 4. In particular, every linklessly embeddable graph is $(4,440)$-choosable. 
A graph is knotlessly embeddable if it has an embedding in $\mathbb{R}^{3}$ in which every cycle forms a trivial knot; see [70] for a survey. Knotlessly embeddable graphs form a minor-closed class whose minimal excluded minors include $K_{7}$ and $K_{3,3,1,1}$ [22, 32]. More than 260 minimal excluded minors are known [35], but the full list of minimal excluded minors is unknown. Since knotlessly embeddable graphs exclude $K_{7}$ minors, they are 8-colourable [1, 45]. Mader [61] proved that $K_{7}$-minor-free graphs have average degree less than 10, which implies they are 9-degenerate and thus 10 -choosable. It is open whether $K_{7}$-minor-free graphs or knotlessly embeddable graphs are 6-colourable or 7-choosable [4]. A graph is 2-apex if deleting at most two vertices makes it planar. Blain et al. [6] and Ozawa and Tsutsumi [67] proved that every 2-apex graph is knotlessly embeddable. Since every block of $G(5, N)$ is 2-apex, $G(5, N)$ is knotlessly embeddable. By Lemma 2.4, the class of knotlessly embeddable graphs has defective chromatic number at least 5 . Since $K_{3,3,1,1}$ is a minor of $K_{5,3}^{*}$, knotlessly embeddable graphs do not contain a $K_{5,3}^{*}$ subgraph. Since knotlessly embeddable graphs have average degree less than 10, Theorem 2.3 implies the following result.

Theorem 6.2. The class of knotlessly embeddable graphs has defective chromatic number and defective choice number 5. In particular, every knotlessly embeddable graph is $(5,660)$-choosable.

6.2. Excluded Complete and Complete Bipartite Minors. Now consider graphs excluding a given complete graph as a minor. Edwards et al. [31] proved that the class of $K_{s+1}$-minor-free graphs has defective chromatic-number $s$, which is a weakening of Hadwiger's conjecture. They also noted that the same method proves the same result for $K_{s+1}$-topological minor-free graphs. We have the following choosability versions of these results.

Theorem 6.3. For each integer $s \geqslant 2$, the class of $K_{s+1}$-minor-free graphs has defective chromatic-number $s$ and defective choice number $s$. In particular, if $\delta$ is the maximal density of a $K_{s+1}$-minor-free graph, then every $K_{s+1}$-minor-free graph is $(s,\lfloor\delta(2 \delta-s+1)\rfloor-s+1)$-choosable. The same result holds replacing "minor" by "topological minor".

The lower bound in Theorem 6.3 follows from Lemma 2.4. The upper bound follows from Theorem 2.3 with $t=1$ since $K_{s, 1}^{*}$ has a $K_{s+1}$-topological-minor. Indeed, in the $t=1$ case, the proof of Theorem 2.3 is the same as the proof of Edwards et al. [31] with essentially the same degree bound. For $K_{s+1}$-minorfree graphs, Kostochka [53, 54] and Thomason [82, 83] proved that the maximum density $\delta=\Theta(s \sqrt{\log s})$, and thus every $K_{s+1}$-minor-free graph is $\left(s, O\left(s^{2} \log s\right)\right)$ choosable. For $K_{s+1}$-topological-minor-free graphs, Bollobás and Thomason [7] and Komlós and Szemerédi [52] proved that the maximum density $\delta=\Theta\left(s^{2}\right)$, and thus every $K_{s+1}$-topological-minor-free graph is $\left(s, O\left(s^{4}\right)\right)$-choosable. Finally, note that 
for $K_{s+1}$-minor-free graphs, choice number and defective choice number substantially differ, since Barát et al. [4] constructed $K_{s+1}$-minor-free graphs that are not $\frac{4}{3}(s-1)$-choosable (for infinitely many $s$ ).

Now we deduce a theorem for the class of graphs with no $K_{s, t}$ topological minor.

Theorem 6.4. For integers $t \geqslant s \geqslant 1$, the defective chromatic number and the defective choice number of the class of $K_{s, t}$ topological minor-free graphs are equal to s. In particular, every $K_{s, t}$ topological minor-free graph is $\left(s, 2^{O(s \log t)}\right)$-choosable.

Proof. The lower bound follows from Lemma 2.4. since $G(s, N)$ contains no $K_{s, t}$ topological minor. For the upper bound, Reed and Wood [71] noted that a method of Diestel [27], which is based on a result about linkages due to Thomas and Wollan [81], shows that for every graph $H$ with $p$ vertices and $q$ edges, every graph with average degree at least $4 p+20 q$ contains $H$ as a topological minor. Thus every $K_{s, t}$-topological-minor-free $\operatorname{graph} G$ has $\operatorname{mad}(G) \leqslant 20 s t+4(s+t) \leqslant 4(5 s+2) t$ and $\widetilde{\nabla}_{1 / 2}(G) \leqslant 2(5 s+2) t$. By Theorem 2.3, $G$ is $(s, d)$-choosable, where $d:=$ $\left\lfloor N_{1}(s, t, 4(5 s+2) t, 4(5 s+2) t)-s+1\right\rfloor$, which is in $2^{O(s \log t)}$.

Note that Theorem 6.4 implies and is more general than Theorem 6.3, since $K_{s, t}$ contains $K_{s+1}$ as a minor (for $t \geqslant s$ ). For $K_{s, t}$-minor-free graphs, the degree bound in Theorem 6.4 can be improved by using known results on the extremal function for $K_{s, t}$-minor-free graphs [36, 55, 59].

6.3. Colin de Verdière Parameter. The Colin de Verdière parameter $\mu(G)$ is an important graph invariant introduced by Colin de Verdière [20, 21]; see [78, 84, 85] for surveys. It is known that $\mu(G) \leqslant 1$ if and only if $G$ is a forest of paths, $\mu(G) \leqslant 2$ if and only if $G$ is outerplanar, $\mu(G) \leqslant 3$ if and only if $G$ is planar, and $\mu(G) \leqslant 4$ if and only if $G$ is linklessly embeddable. A famous conjecture of Colin de Verdière [20] states that $\chi(G) \leqslant \mu(G)+1$ (which implies the 4-colour theorem, and is implied by Hadwiger's Conjecture). For defective colourings one fewer colour suffices.

Theorem 6.5. For $k \geqslant 1$, the defective chromatic number and the defective choice number of the class of graphs $G$ with $\mu(G) \leqslant k$ are equal to $k$. In particular, every graph $G$ with $\mu(G) \leqslant k$ is $\left(k, 2^{O(k \log \log k)}\right)$-choosable.

Proof. Graphs with $\mu(G) \leqslant k$ form a minor-closed class [20, 21]. van der Holst et al. [85] proved that $\mu\left(K_{s, t}\right)=s+1$ for $t \geqslant \max \{s, 3\}$. Thus, if $\mu(G) \leqslant k$ then $G$ contains no $K_{k, \max (k, 3)} \operatorname{minor}$, and $\operatorname{mad}(G) \leqslant 2 \widetilde{\nabla}_{1 / 2}(G) \leqslant O(k \sqrt{\log k})$. Theorem 2.3 with $s=k$ and $t=\max \{k, 3\}$ implies that $G$ is $\left(k, 2^{O(k \log \log k)}\right)$-choosable. Now we prove the lower bound. van der Holst et al. [85] proved that $\mu(G)$ equals the maximum of $\mu\left(G^{\prime}\right)$, taken over the components $G^{\prime}$ of $G$, and if $G$ has a dominant vertex $v$, then $\mu(G)=\mu(G-v)+1$. It follows that $\mu(G(k, N))=k$ for $N \geqslant 2$. Lemma 2.4 then implies that the class of graphs with $\mu(G) \leqslant k$ has defective chromatic number and defective choice number at least $k$.

Theorem 6.5 generalises Theorem 6.1 which corresponds to the case $k=4$. 
6.4. $H$-Minor-Free Graphs. This section considers, for an arbitrary graph $H$, the defective chromatic number of the class of $H$-minor-free graphs, which we denote by $f(H)$. That is, $f(H)$ is the minimum integer such that there exists an integer $d(H)$ such that every $H$-minor-free graph has a $(f(H), d(H))$-colouring. Obviously, $f$ is minor-monotone: if $H^{\prime}$ is a minor of $H$, then every $H^{\prime}$-minor-free graph is $H$-minor-free, and thus $f\left(H^{\prime}\right) \leqslant f(H)$.

A set $S$ of vertices in a graph $H$ is a vertex cover if $E(H-S)=\emptyset$. Let $\tau(H)$ be the minimum size of a vertex cover in $H$, called the vertex cover number of $H$. The tree-depth of a connected graph $H$, denoted by $\operatorname{td}(H)$, is the minimum height of a rooted tree $T$ such that $H$ is a subgraph of the closure of $T$. Here the closure of $T$ is obtained from $T$ by adding an edge between every ancestor and descendent in $T$. The height of a rooted tree is the maximum number of vertices on a root-to-leaf path. The tree-depth of a disconnected graph $H$ is the maximum tree-depth of the connected components of $H$.

Proposition 6.6. For every graph $H$,

$$
\operatorname{td}(H)-1 \leqslant f(H) \leqslant \tau(H) .
$$

Proof. Obviously, $H$ is a minor of $K_{\tau(H),|V(H)|-\tau(H)}^{*}$. Thus every $H$-minor-free graph is $K_{\tau(H),|V(H)|-\tau(H)}^{*}$ free. By Theorem 2.3, $f(H) \leqslant \tau(H)$.

We now establish the lower bound on $f(H)$. Observe that $G(s, N)$ is the closure of the complete $(N+1)$-ary tree of height $s$, and $G(s, N)$ has tree-depth at most $s$. Since tree-depth is minor-monotone [65], every minor of $G(s, N)$ has tree-depth at most $s$. Thus a graph $H$ is not a minor of $G(\operatorname{td}(H)-1, N)$. By Lemma 2.4, every $(\operatorname{td}(H)-2)$-colouring of $G(\operatorname{td}(H)-1, N)$ has a colour class that induces a subgraph with maximum degree at least $N$. Thus $f(H) \geqslant \operatorname{td}(H)-1$.

The lower and upper bounds in Proposition 6.6 match in some important cases, like $H=K_{t}$ or $H=K_{s, t}$ (or $H=K_{s, t}^{*}$ ). The Petersen graph $P$ is an example where they do not match. Proposition 6.6 implies $f(P) \in\{5,6\}$. On the other hand, every $P$-minor-free graph is 9-colourable [41] and this is best possible since $K_{9}$ is $P$-minor-free. So our upper bound improves the obvious bound deduced from chromatic number. Paths provide an interesting example where the bounds in Proposition 6.6 are far apart. In particular, for a path of order $2^{t}-1$, Proposition 6.6 gives

$$
t-1 \leqslant f\left(P_{2^{t}-1}\right) \leqslant 2^{t-1}-1 .
$$

It is easy to characterise the graphs with $f(H)=1$, in which case the lower and upper bounds in Proposition 6.6 are equal.

Proposition 6.7. $f(H)=1$ if and only if $H$ is a star plus some isolated vertices.

Proof. Say $H$ is a $k$-leaf star plus $\ell$ isolated vertices. Consider a graph $G$. If $G$ has maximum degree at most $k-1$, then $G$ is $(1, k-1)$-colourable. If $G$ has at most $k+\ell$ vertices, then $G$ is $(1, k+\ell-1)$-colourable. Otherwise, $G$ has maximum degree at 
least $k$ and has at least $k+1+\ell$ vertices, in which case $G$ contains $H$ as a minor. Thus every $H$-minor-free graph is $(1, k+\ell-1)$-colourable, and $f(H)=1$. Conversely, say $H$ is not a star plus some isolated vertices. Then $H$ has two disjoint edges. For each integer $d$, the $(d+1)$-leaf star has no $H$-minor and is not $(1, d)$-colourable. Thus $f(H) \geqslant 2$.

The upper bound in Proposition 6.6 is not tight in general. For example, if $H$ is the $k$-ary tree of height 3 , then $\tau(H)=k$ but $f(H)=2$ (as proved in Theorem 6.9 below). These observations lead to the following conjecture:

Conjecture 6.8. $f(H)=\operatorname{td}(H)-1$ for every graph $H$, unless $H$ has distinct connected components $H_{1}$ and $H_{2}$ with $\operatorname{td}(H)=\operatorname{td}\left(H_{1}\right)=\operatorname{td}\left(H_{2}\right)$, in which case $f(H)=\operatorname{td}(H)$.

We now explain the necessity of the exception in Conjecture 6.8. Suppose $H$ has connected components $H_{1}$ and $H_{2}$ with $\operatorname{td}(H)=\operatorname{td}\left(H_{1}\right)=\operatorname{td}\left(H_{2}\right)=s$. If $H$ is a minor of $G(s, n)$, then only one of $H_{1}$ and $H_{2}$ can use the root vertex of $G(s, n)$, implying one of $H_{1}$ and $H_{2}$ is a minor of $G(s-1, n)$, which contradicts the treedepth assumption. Thus, $H$ is not a minor of $G(s, n)$. By Lemma 2.4, the class of $H$-minor-free graphs has defective chromatic number at least $s=\operatorname{td}(H)$.

Proposition 6.7 confirms Conjecture 6.8 when $f(H)=1$. We now prove the first non-trivial case.

Theorem 6.9. For every graph $H$ with tree-depth 3 and with at most one component of tree-depth 3, the defective chromatic number of the class of $H$-minor-free graphs equals 2.

Proof. The lower bound is proved above. For the upper bound, since at most one component of $H$ has tree-depth $3, H$ is a subgraph of $G(2, k)$ for some integer $k \leqslant|V(H)|$. By Lemma 6.10 below with $\ell=k$, every $G(2, k)$-minor-free graph is $(2, d)$-colourable, for some increasing function $d=d(k)$. Every $H$-minor-free graph is $G(2, k)$-minor-free. Since $k \leqslant|V(H)|$, every $H$-minor-free graph is $(2, d)$-colourable, where $d=d(k) \leqslant d(|V(H)|)$.

Lemma 6.10. Let $H$ be the graph obtained from $\ell$ disjoint copies of $K_{1, k}$ by adding one dominant vertex, for some $\ell \geqslant 2$ and $k \geqslant 1$ (as illustrated in Figure 2). Then every $H$-minor-free graph $G$ is $\left(2, O\left(\ell^{10} k^{3}\right)\right)$-colourable.

Proof. Since $H$ is 2-degenerate, there exists $\delta<7(\ell k+\ell+1)$ such that every $H$ minor-free graph has average degree at most $\delta$ by a result of Reed and Wood [71, Lemma 3.3]. Let $r:=\left(\begin{array}{c}\ell^{2}-1 \\ 2\end{array}\right)(k+1)+\ell^{2}+\ell$. Let $X$ be the set of vertices $v \in V(G)$ such that $\left|N_{G}(v) \cap N_{G}(w)\right| \geqslant r$ for some vertex $w \in V(G) \backslash\{v\}$. Note that $w$ is also in $X$. Let $Q$ be the graph with vertex set $V(G)$ where $v w \in E(Q)$ whenever $\left|N_{G}(v) \cap N_{G}(w)\right| \geqslant r$. For each edge $e=v w \in E(Q)$, let $N(e):=N_{G}(v) \cap N_{G}(w)$. Thus $|N(e)| \geqslant r$. Let $Y:=V(G) \backslash X$.

Claim 1. Q has maximum degree less than $\ell$. 


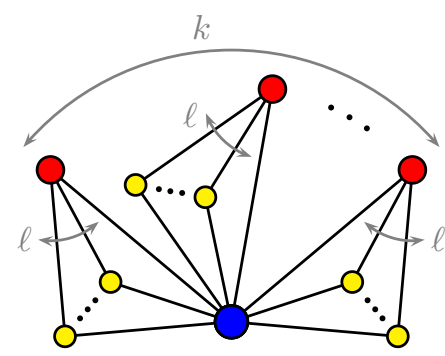

Figure 2. The graph $H$.

Proof. Suppose on the contrary that some vertex $v$ in $Q$ is adjacent to distinct vertices $v_{1}, \ldots, v_{\ell}$ in $Q$. For $i=1,2, \ldots, \ell$, choose $k+1$ common neighbours of $v$ and $v_{i}$ in $G$ that have not already been chosen and are different from $v, v_{1}, \ldots, v_{\ell}$. This is possible, since $v$ and $v_{i}$ have $r \geqslant(k+1) \ell+\ell-1$ common neighbours in $G$. For each $i \in[\ell]$, contract the edge between $v_{i}$ and one of the chosen common neighbours of $v$ and $v_{i}$. The chosen vertices along with $v, v_{1}, \ldots, v_{\ell}$ form $H$ as a minor of $G$, which is a contradiction.

Claim 2. If $R$ is a set of more than $\ell(\ell-1)$ vertices in $X$, then $Q$ contains an $\ell$-edge matching, each edge of which has at least one endpoint in $R$.

Proof. Let $Z$ be the subgraph of $Q$ induced by $R \cup N_{Q}(R)$. Label vertices in $R$ red and vertices in $N_{Q}(R) \backslash R$ blue. If $\Delta$ is the maximum degree of $Z$, then $\Delta \leqslant \ell-1$ by Claim 1. The number of red vertices is $|R|>(\Delta+1)(\ell-1)$. Every vertex in $R$ is in $X$ and thus has a neighbour in $Q$, which is in $N_{Q}(R)$. Hence $Z$ has no red isolated vertex. Let $Z^{\prime}$ be an edge-minimal spanning subgraph of $Z$ with no red isolated vertex. By minimality, each edge of $Z^{\prime}$ has a red endpoint with degree 1 . Thus each component of $Z^{\prime}$ is either a blue isolated vertex, a redblue edge, or a star with all its leaves coloured red. Since each component of $Z^{\prime}$ has at most $\Delta+1$ red vertices, and there are strictly greater than $(\Delta+1)(\ell-1)$ red vertices, $Z^{\prime}$ contains at least $\ell$ non-singleton components. Let $v_{1} w_{1}, \ldots, v_{\ell} w_{\ell}$ be a matching obtained by choosing one edge from each non-singleton component of $Z^{\prime}$, where $v_{1}, \ldots, v_{\ell}$ are red and thus in $R$.

Claim 3. Every vertex is adjacent in $G$ to less than $\ell^{2}$ vertices in $X$.

Proof. Suppose on the contrary that $\left|N_{G}(v) \cap X\right| \geqslant \ell^{2}$ for some vertex $v \in V(G)$. If $v \in X$ then let $R:=\left(N_{G}(v) \cap X\right) \backslash N_{Q}(v)$, otherwise let $R:=N_{G}(v) \cap X$. Then $|R|>\ell(\ell-1)$ since $\left|N_{Q}(v)\right| \leqslant \ell-1$ by Claim 1. By Claim 2, $Q$ contains a matching $v_{1} w_{1}, \ldots, v_{\ell} w_{\ell}$, where $v_{1}, \ldots, v_{\ell}$ are in $R$. By construction, each $w_{i} \neq v$. For $i=1,2, \ldots, \ell$, choose $k+1$ common neighbours of $v_{i}$ and $w_{i}$ in $G$ that have not already been chosen and are different from $v, v_{1}, w_{1}, \ldots, v_{\ell}, w_{\ell}$. This is possible, since $v_{i}$ and $w_{i}$ have $r \geqslant \ell(k+1)+2 \ell-1$ common neighbours. For each $i \in[\ell]$, contract the edge $v v_{i}$ into $v$, and contract the edge between $w_{i}$ and one 
of the chosen common neighbours of $v_{i}$ and $w_{i}$. The chosen vertices along with $v, w_{1}, \ldots, w_{\ell}$ form $H$ as a minor of $G$, which is a contradiction.

Let $G^{\prime}$ be the graph obtained from $G$ as follows. For each component $C$ of $Q$, identify $V(C)$ into one vertex, and delete resulting loops and parallel edges. Each vertex of $G^{\prime}$ corresponds to a component of $Q$.

The final step of this proof applies Theorem 2.3 with $s=2$ to obtain a defective 2-colouring of $G^{\prime}$, from which we obtain a defective 2-colouring of $G$. To apply Theorem 2.3 we show that $G^{\prime}$ has no large $K_{2, t}$ subgraph, has bounded $\widetilde{\nabla}_{1 / 2}$, and (thus) bounded average degree.

Consider a $K_{2, t}$ subgraph in $G^{\prime}$. There are distinct components $C, D, A_{1}, \ldots, A_{t}$ of $Q$, such that for $i \in[t]$, some vertex in $A_{i}$ is adjacent in $G$ to some vertex in $C$, and some vertex in $A_{i}$ is adjacent in $G$ to some vertex in $D$. Note each $A_{i}$ is either a single-vertex component of $Q$ contained in $Y$ or is contained in $X$ with at least two vertices.

Claim 4. $\left|\left\{i \in[t]: A_{i} \subseteq X\right\}\right|<\ell^{2}$

Proof. Suppose on the contrary and without loss of generality that $A_{1}, \ldots, A_{\ell^{2}} \subseteq$ $X$. The component $C$ is not a single vertex, as otherwise, this vertex would have at least $\ell^{2}$ neighbours in $X$ contradicting Claim 3. Thus $C$ is contained in $X$ and has at least two vertices. For each $i \in[\ell]$, let $v_{i}$ be a vertex in $A_{i}$ adjacent to a vertex in $C$. Since $v_{i}$ is in $A_{i} \subseteq X$, there is an edge $e_{i}=u_{i} v_{i} \in E(Q)$ and thus $u_{i}$ is also in $A_{i}$. Note that $u_{1}, \ldots, u_{\ell}$ are distinct since they belong to different components $A_{i}$. Let $E(C)$ be the set of edges of $Q$ between vertices in $C$. Construct a bipartite graph $B$ with colour classes

$$
B_{1}:=E(C) \cup\left\{e_{i}^{j}: i \in[\ell], j \in[k+1]\right\} \text { and } B_{2}:=Y,
$$

where the vertex corresponding to each $f \in E(C)$ is adjacent to each vertex in $N(f) \backslash X$, and similarly the vertex $e_{i}^{j}$ is adjacent to each vertex in $N\left(e_{i}\right) \backslash X$ for each $i \in[\ell]$ and $j \in[k+1]$. The endpoints of each edge in $Q$ have at least $r$ common neighbours in $G$, at most $\ell^{2}-1$ of which are in $X$ by Claim 3 . Thus, in $B$, every vertex in $B_{1}$ has degree at least $r-\ell^{2}+1$, and every vertex in $B_{2}$ has degree at most $\left(\begin{array}{c}\ell^{2}-1 \\ 2\end{array}\right)(k+1)$ by Claim 3. Consider a subset $S \subseteq B_{1}$. The number of edges between $S$ and $N_{B}(S)$ is at least $\left(r-\ell^{2}+1\right)|S|$ and at most $\left(\begin{array}{c}\ell^{2}-1 \\ 2\end{array}\right)(k+1)\left|N_{B}(S)\right|$, implying $\left|N_{B}(S)\right| \geqslant|S|$ since $r-\ell^{2}+1 \geqslant\left(\begin{array}{c}\ell^{2}-1 \\ 2\end{array}\right)(k+1)$. By Hall's Theorem, $B$ contains a matching with every vertex in $B_{1}$ matched. For $i \in[\ell]$ and $j \in[k+1]$, let $x_{i}^{j}$ be the vertex in $B_{2}$ matched with $e_{i}^{j}$. Then $x_{i}^{j}$ is a common neighbour of $u_{i}$ and $v_{i}$ in $G$. For each edge $f \in E(C)$, let $x_{f}$ be the vertex in $B_{2}$ matched with $f$. Then $x_{f}$ is a common neighbour of the endpoints of $f$ in $G$. All these $x$-vertices are distinct and are contained in $Y$. Hence $V(C) \cup\left\{x_{f}: f=p q \in E(C)\right\} \cup\left\{v_{1}, v_{2}, \ldots, v_{\ell}\right\}$ induces a connected subgraph of $G-\left(\left\{u_{1}, u_{2}, \ldots, u_{\ell}\right\} \cup\left\{x_{i}^{j}: i \in[\ell], j \in[k+1]\right\}\right)$; contract this connected subgraph into a vertex $z$. Now $z$ is adjacent to $x_{i}^{j}$ for each $i \in[\ell]$ and $j \in[k+1]$. Finally, 
contract the edge $u_{i} x_{i}^{k+1}$ into $u_{i}$, for each $i \in[\ell]$. Now $z$ is adjacent to $u_{1}, \ldots, u_{\ell}$, and $x_{i}^{1}, \ldots, x_{i}^{k}$ are common neighbours of $z$ and $u_{i}$. Hence $H$ is a minor of $G$, which is a contradiction.

Claim 5. $\left|\left\{i \in[t]: A_{i} \subseteq Y\right\}\right| \leq \ell^{2}(\ell-1)^{2}(r-1)$.

Proof. Define $Z:=\bigcup\left\{A_{i}: i \in[t], A_{i} \subseteq Y\right\}$. Note that $|Z|=\left|\left\{i \in[t]: A_{i} \subseteq Y\right\}\right|$ because $\left|A_{i}\right|=1$ if $A_{i} \subseteq Y$. Let $C^{\prime}$ be the set of vertices in $C$ with some neighbour in $Z$. Let $D^{\prime}$ be the set of vertices in $D$ with some neighbour in $Z$. For $v \in V\left(C^{\prime}\right)$ and $w \in V\left(D^{\prime}\right)$, less than $r$ vertices are common neighbours of $v$ and $w$ (since $v w \notin E(Q))$. Thus $|Z| \leq\left|C^{\prime}\right|\left|D^{\prime}\right|(r-1)$, and we are done if $\left|C^{\prime}\right| \leq \ell(\ell-1)$ and $\left|D^{\prime}\right| \leq \ell(\ell-1)$.

Suppose for the sake of contradiction and without loss of generality that $\left|C^{\prime}\right|>$ $\ell(\ell-1)$. Then $V(C) \subseteq X$ since $\ell(\ell-1) \geqslant 2$. By Claim 2 with $R=C^{\prime}$, there is a matching $e_{1}, \ldots, e_{\ell}$ in $Q$, where $e_{i}=v_{i} u_{i}$ and $v_{i} \in C^{\prime}$ for each $i \in[\ell]$. For $i \in[\ell]$, let $a_{i}$ be a (not necessarily distinct) neighbour of $v_{i}$ in $Z$. Let $E(D)$ be the set of edges in $Q$ between vertices in $D$. Construct a bipartite graph $B$ with colour classes

$$
\begin{aligned}
& B_{1}:=E(D) \cup\left\{e_{i}^{j}: i \in[\ell], j \in[k+1]\right\} \text { and } \\
& B_{2}:=Y \backslash\left(\left\{a_{1}, \ldots, a_{\ell}\right\} \cup V(D)\right),
\end{aligned}
$$

where the vertex in $B_{1}$ corresponding to each edge $f \in E(D)$ is adjacent to each vertex in $N(f) \backslash\left(X \cup V(D) \cup\left\{a_{1}, \ldots, a_{\ell}\right\}\right)$, and similarly the vertex in $B_{1}$ corresponding to each edge $e_{i}^{j}$ is adjacent to each vertex in $N\left(e_{i}\right) \backslash(X \cup V(D) \cup$ $\left.\left\{a_{1}, \ldots, a_{\ell}\right\}\right)$. Note that $|Y \cap V(D)| \leq 1$ and if $E(D) \neq \emptyset$, then $V(D) \subseteq X$. The endpoints of each edge in $Q$ have at least $r$ common neighbours in $G$, at most $\left(\ell^{2}-1\right)+\ell+1$ of which are in $X \cup V(D) \cup\left\{a_{1}, \ldots, a_{\ell}\right\}$ by Claim 3. Thus, in $B$, every vertex in $B_{1}$ has degree at least $r-\ell^{2}-\ell$, and every vertex in $B_{2}$ has degree at most $\left(\begin{array}{c}\ell^{2}-1 \\ 2\end{array}\right)(k+1)$ by Claim 3. Consider a subset $S \subseteq B_{1}$. The number of edges between $S$ and $N_{B}(S)$ is at least $\left(r-\ell^{2}-\ell\right)|S|$ and at most $\left(\begin{array}{c}\ell^{2}-1 \\ 2\end{array}\right)(k+1)\left|N_{B}(S)\right|$, implying $\left|N_{B}(S)\right| \geqslant|S|$ since $r-\ell^{2}-\ell \geqslant\left(\begin{array}{c}\ell^{2}-1 \\ 2\end{array}\right)(k+1)$. By Hall's Theorem, $B$ contains a matching with every vertex in $B_{1}$ matched.

For $f \in E(D)$, let $x_{f}$ be the vertex in $B_{2}$ matched with $f$ and for each $i \in[\ell]$ and $j \in[k+1]$, let $x_{i}^{j}$ be the vertex in $B_{2}$ matched with $e_{i}^{j}$. Then $x_{f}$ is a common neighbour (in $G$ ) of the endpoints of $f$ and $x_{i}^{j}$ is a common neighbour of $u_{i}$ and $v_{i}$ in $G$. Note that all these $x$-vertices are distinct and are in $V(G) \backslash(X \cup V(D) \cup$ $\left.\left\{a_{1}, \ldots, a_{\ell}\right\}\right)$. Each vertex $a_{i}$ has a neighbour in $D$. Hence $\left\{a_{1}, \ldots, a_{\ell}, v_{1}, \ldots, v_{\ell}\right\} \cup$ $V(D) \cup\left\{x_{f}: f \in E(D)\right\}$ induces a connected subgraph of $G-\left(\left\{u_{1}, u_{2}, \ldots, u_{\ell}\right\} \cup\right.$ $\left.\left\{x_{i}^{j}: i \in[\ell], j \in[k+1]\right\}\right)$. Contract this connected subgraph into a vertex $z$. Now $z$ is adjacent to $x_{i}^{j}$ for each $i \in[\ell]$ and $j \in[k+1]$. Finally, contract the edge $u_{i} x_{i}^{k+1}$ into $u_{i}$, for each $i \in[\ell]$. Now $z$ is adjacent to $u_{1}, \ldots, u_{\ell}$, and $x_{i}^{1}, \ldots, x_{i}^{k}$ are common neighbours of $z$ and $u_{i}$ for each $i \in[\ell]$. Hence $H$ is a minor of $G$, which is a contradiction. 
Claims 4 and 5 show that $t<\ell^{2}+\ell^{2}(\ell-1)^{2}(r-1) \leq \ell^{2}(\ell-1)^{2} r$. That is, $G^{\prime}$ has no $K_{2, \ell^{2}(\ell-1)^{2} r}$ subgraph.

Claim 6. $\widetilde{\nabla}_{1 / 2}\left(G^{\prime}\right) \leqslant \delta / 2+\ell-1$.

Proof. Suppose that a $(\leqslant 1)$-subdivision of some graph $G^{\prime \prime}$ is a subgraph of $G^{\prime}$. Let $X^{\prime \prime}$ be the set of vertices of $G^{\prime \prime}$ that arise from components of $Q$ contained in $X$.

Assume for contradiction that some vertex in $G^{\prime \prime}$ has at least $\ell$ neighbours in $X^{\prime \prime}$. That is, there are distinct components $C, C_{1}, \ldots, C_{\ell}$ of $Q$, such that for each $i \in[\ell], C_{i}$ is a non-singleton component of $Q$ contained in $X$, and there exists an edge joining a vertex of $C$ to a vertex of $C_{i}$ in $G$, or a component $D_{i}$ of $Q$ having a neighbour of $C$ and a neighbour of $C_{i}$ in $G$. If $C$ and $C_{i}$ are joined by an edge in $G$, then let $D_{i}:=C$ (for convenience). Note that $C$ might be a singleton component of $Q$ contained in $Y$ or a non-singleton component contained in $X$, and similarly for $D_{1}, \ldots, D_{\ell}$, but $C_{1}, \ldots, C_{\ell}$ are non-singleton components of $Q$. Let $Y^{\prime}=Y \cap\left(V(C) \cup V\left(D_{1}\right) \cup \cdots \cup V\left(D_{\ell}\right)\right)$. Then $\left|Y^{\prime}\right| \leqslant \ell+1$. For each $i \in[\ell]$, let $v_{i}$ be a vertex in $C_{i}$ adjacent to some vertex in $V(C) \cup V\left(D_{i}\right)$. Since $v_{i}$ is in $X$, there is an edge $e_{i}=u_{i} v_{i} \in E(Q)$ and thus $u_{i}$ is also in $C_{i}$. Construct a bipartite graph $B$ with colour classes

$$
\begin{aligned}
& B_{1}:=E(C) \cup E\left(D_{1}\right) \cup \cdots \cup E\left(D_{\ell}\right) \cup\left\{e_{i}^{j}: i \in[\ell], j \in[k+1]\right\} \text { and } \\
& B_{2}:=V(G) \backslash\left(X \cup Y^{\prime}\right),
\end{aligned}
$$

where the vertex corresponding to each edge $f \in E(C) \cup E\left(D_{1}\right) \cup \cdots \cup E\left(D_{\ell}\right)$ is adjacent to each vertex in $N(f) \backslash\left(X \cup Y^{\prime}\right)$, and similarly the vertex $e_{i}^{j}$ is adjacent to each vertex in $N\left(e_{i}\right) \backslash\left(X \cup Y^{\prime}\right)$. The endpoints of each edge in $Q$ have at least $r$ common neighbours in $G$, at most $\ell^{2}-1+\left|Y^{\prime}\right|$ of which are in $X \cup Y^{\prime}$ by Claim 3 . Thus, in $B$, every vertex in $B_{1}$ has degree at least $r-\left(\ell^{2}-1+\left|Y^{\prime}\right|\right) \geqslant r-\ell^{2}-\ell$, and every vertex in $B_{2}$ has degree at most $\left(\begin{array}{c}\ell^{2}-1 \\ 2\end{array}\right)(k+1)$ by Claim 3 . Consider a subset $S \subseteq B_{1}$. The number of edges between $S$ and $N_{B}(S)$ is at least $\left(r-\ell^{2}-\ell\right)|S|$ and at most $\left(\begin{array}{c}\ell^{2}-1 \\ 2\end{array}\right)(k+1)\left|N_{B}(S)\right|$, implying $\left|N_{B}(S)\right| \geqslant|S|$ since $r-\ell^{2}-\ell \geqslant\left(\begin{array}{c}\ell^{2}-1 \\ 2\end{array}\right)(k+1)$.

By Hall's Theorem, $B$ contains a matching with every vertex in $B_{1}$ matched. For $i \in[\ell]$ and $j \in[k+1]$, let $x_{i}^{j}$ be the vertex in $B_{2}$ matched with $e_{i}^{j}$. Then $x_{i}^{j}$ is a common neighbour of $u_{i}$ and $v_{i}$ in $G$. For each edge $f \in E(C) \cup E\left(D_{1}\right) \cup$ $\cdots \cup E\left(D_{\ell}\right)$, let $x_{f}$ be the vertex in $B_{2}$ matched with $f$. Then $x_{f}$ is a common neighbour (in $G$ ) of the endpoints of $f$. All these $x$-vertices are distinct and are contained in $V(G) \backslash\left(X \cup Y^{\prime}\right)$. Hence

$V(C) \cup V\left(D_{1}\right) \cdots \cup V\left(D_{\ell}\right) \cup\left\{v_{1}, v_{2}, \ldots, v_{\ell}\right\}$

$$
\cup\left\{x_{f}: f \in E(C) \cup E\left(D_{1}\right) \cup \cdots \cup E\left(D_{\ell}\right)\right\}
$$

induces a connected subgraph of $G-\left(\left\{u_{1}, u_{2}, \ldots, u_{\ell}\right\} \cup\left\{x_{i}^{j}: i \in[\ell], j \in[k+1]\right\}\right)$; contract this connected subgraph into a vertex $z$. Now $z$ is adjacent to $x_{i}^{j}$ for each $i \in[\ell]$ and $j \in[k+1]$. Finally, contract the edge $u_{i} x_{i}^{k+1}$ into $u_{i}$ for each 
$i \in[\ell]$. Now $z$ is adjacent to $u_{1}, \ldots, u_{\ell}$, and $x_{i}^{1}, \ldots, x_{i}^{k}$ are common neighbours of $z$ and $u_{i}$ for each $i \in[\ell]$. Hence $H$ is a minor of $G$. This contradiction proves that each vertex in $G^{\prime \prime}$ has less than $\ell$ neighbours in $X^{\prime \prime}$.

Thus $G^{\prime \prime}$ contains at most $(\ell-1)\left|V\left(G^{\prime \prime}\right)\right|$ edges with at least one endpoint in $X^{\prime \prime}$. Since $G^{\prime \prime}-X^{\prime \prime}$ is a subgraph of $G$, the average degree of $G^{\prime \prime}-X^{\prime \prime}$ is at most $\delta$, and $\left|E\left(G^{\prime \prime}-X^{\prime \prime}\right)\right| \leq \delta\left|Y^{\prime}\right| / 2$. In total, $\left|E\left(G^{\prime \prime}\right)\right| \leqslant \delta\left|Y^{\prime}\right| / 2+(\ell-1)\left|V\left(G^{\prime \prime}\right)\right| \leqslant$ $(\delta / 2+\ell-1)\left|V\left(G^{\prime \prime}\right)\right|$, and $\widetilde{\nabla}_{1 / 2}\left(G^{\prime}\right) \leqslant \delta / 2+\ell-1$.

Note that Claim 6 implies $\operatorname{mad}(G)=2 \widetilde{\nabla}_{0}(G) \leqslant 2 \widetilde{\nabla}_{1 / 2}(G) \leqslant \delta+2 \ell-2$. By Theorem 2.3, $G^{\prime}$ is $\left(2, d^{\prime}\right)$-colourable, where

$$
d^{\prime}=\left\lfloor N_{1}\left(2, \ell^{2}(\ell-1)^{2} r, \delta+2 \ell-2, \delta+2 \ell-2\right)\right\rfloor-1 .
$$

Colour each vertex $v$ of $G$ by the colour assigned to the vertex of $G^{\prime}$ corresponding to the component of $Q$ containing $v$. Then, for each vertex $v$ of $C$, the number of neighbours of $v$ having the same colour as $v$ is at most $d^{\prime}+\ell^{2}-1$, because $v$ has at most $\ell^{2}-1$ neighbours in $X$ by Claim 3 and at most $d^{\prime}$ neighbours of the same colour in $Y$. Therefore $G$ is $\left(2, d^{\prime}+\ell^{2}-1\right)$-colourable. We now estimate the degree bound. We have $r \leqslant O\left(\ell^{4} k\right)$ and $\delta+2 \ell-2 \leqslant O(\ell k)$. Thus $d^{\prime} \leqslant O\left((\delta+2 \ell)^{2} \ell^{2}(\ell-1)^{2} r\right) \leqslant$ $O\left(\ell^{10} k^{3}\right)$. Therefore $G$ is $\left(2, O\left(\ell^{10} k^{3}\right)\right)$-colourable.

Our final result provides further evidence for Conjecture 6.8. It concerns graphs that exclude a fixed tree as a subgraph.

Proposition 6.11. Let $T$ be a tree with $n \geqslant 2$ vertices and radius $r \geqslant 1$. Then every graph containing no $T$ subgraph is $(r, n-2)$-colourable.

Proof. For $i=1,2, \ldots, r-1$, let $V_{i}$ be the set of vertices $v \in V(G) \backslash\left(V_{1} \cup \cdots \cup V_{i-1}\right)$ that have at most $n-2$ neighbours in $V(G) \backslash\left(V_{1} \cup \cdots \cup V_{i-1}\right)$. Let $V_{r}:=V(G) \backslash$ $\left(V_{1} \cup \cdots \cup V_{r-1}\right)$. Then $V_{1} \cup \cdots \cup V_{r}$ is a partition of $V(G)$. For $i \in[1, r-1]$, by construction, $G\left[V_{i}\right]$ has maximum degree at most $n-2$, as desired. Suppose that $G\left[V_{r}\right]$ has maximum degree at least $n-1$. We now show that $T$ is a subgraph of $G$, where each vertex $v$ of $T$ is mapped to a vertex $v^{\prime}$ of $G$. Let $x$ be the centre of $T$. Map the vertices of $T$ to vertices in $G$ in order of their distance from $x$ in $T$, where $x$ is mapped to a vertex $x^{\prime}$ with degree at least $n-1$ in $G\left[V_{r}\right]$. The key invariant is that each vertex $v$ at distance $i$ from $x$ in $T$ is mapped to a vertex $v^{\prime}$ in $V_{r-i+1} \cup \cdots \cup V_{r}$. If $i=0$ then $v=x$ and by assumption, $v^{\prime}$ has at least $n-1$ neighbours in $V_{r}$. If $i \in[1, r-1]$ then by construction, $v^{\prime}$ has at least $n-1$ neighbours in $V_{r-i} \cup \cdots \cup V_{r}$ (otherwise $v^{\prime}$ would be in $V_{r-i}$ ). Thus there are always unmapped vertices in $V_{r-i} \cup \cdots \cup V_{r}$ to choose as the children of $v$. Hence $T$ is a subgraph. This contradiction shows that $G\left[V_{r}\right]$ has maximum degree at least $n-2$, and $G$ is $(r, n-2)$-colourable.

Note that Proposition 6.11 is best possible for the complete binary tree $T$ of radius $r$, which has tree-depth $r+1$ (see [65, Exercise 6.1]). Thus $G(r, N)$ contains no $T$ 
subgraph, and Lemma 2.4 and Proposition 6.11 imply that the defective chromatic number of the class of graphs containing no $T$ subgraph equals $r$.

Note that the behaviour shown in Proposition 6.11 is qualitatively different from the chromatic number of graphs excluding a given tree as a subgraph. Say $T$ is a tree with $n$ vertices. A well known greedy embedding procedure shows that every graph with minimum degree at least $n-1$ contains $T$ as a subgraph. That is, every graph containing no $T$ subgraph is $(n-2)$-degenerate, and is thus $(n-1)$-colourable. This bound is tight since $K_{n-1}$ contains no $T$ subgraph and is $(n-1)$-chromatic. In short, for the class of graphs containing no $T$ subgraph, the chromatic number equals $n-1$, whereas Proposition 6.11 says that the defective chromatic number is at most the radius of $T$.

Conjecture 6.8 suggests similar behaviour for $H$-minor-free graphs. Say $H$ has $n$ vertices. Hadwiger's Conjecture says that the maximum chromatic number of the class of $H$-minor-free graphs equals $n-1$. It is at least $n-1$ since $K_{n-1}$ is $H$-minor-free, and at most $O(n \sqrt{\log n})$ in general. Conjecture 6.8 says that if $H$ is connected, then the defective chromatic number of the class of $H$-minor-free graphs equals the tree-depth of $H$ minus 1 .

\section{REFERENCES}

[1] B. Albar and D. Gonçalves. On triangles in $K_{r}$-minor free graphs. 2013. arXiv: 1304.5468

[2] M. O. Albertson, D. L. Boutin, and E. Gethner. More results on r-inflated graphs: arboricity, thickness, chromatic number and fractional chromatic number. Ars Math. Contemp., 4(1):5-24, 2011.

[3] D. Archdeacon. A note on defective colorings of graphs in surfaces. J. Graph Theory, 11(4):517-519, 1987. doi: 10.1002/jgt.3190110408.

[4] J. Barát, G. Joret, and D. R. Wood. Disproof of the list Hadwiger conjecture. Electron. J. Combin., 18(1):P232, 2011. http://www.combinatorics. org/ojs/index.php/eljc/article/view/v18i1p232.

[5] F. Bernhart and P. C. Kainen. The book thickness of a graph. Journal of Combinatorial Theory, Series B, 27(3):320-331, 1979. doi: 10.1016/0095-8956(79)90021-2.

[6] P. Blain, G. Bowlin, T. Fleming, J. Foisy, J. Hendricks, and J. Lacombe. Some results on intrinsically knotted graphs. J. Knot Theory Ramifications, 16(6): 749-760, 2007. doi: 10.1142/S021821650700552X.

[7] B. Bollobás and A. Thomason. Proof of a conjecture of Mader, Erdős and Hajnal on topological complete subgraphs. European J. Combin., 19(8):883-887, 1998. doi: 10.1006/eujc.1997.0188.

[8] O. V. Borodin. On the total coloring of planar graphs. J. Reine Angew. Math., 394:180-185, 1989. doi:10.1515/crll.1989.394.180.

[9] O. V. Borodin and A. V. Kostochka. Defective 2-colorings of sparse graphs. J. Combin. Theory Ser. B, 104:72-80, 2014. doi:10.1016/j.jctb.2013.10.002. 
[10] O. V. Borodin and D. P. Sanders. On light edges and triangles in planar graphs of minimum degree five. Math. Nachr., 170:19-24, 1994. doi: 10.1002/mana.19941700103.

[11] O. V. Borodin, A. V. Kostochka, N. N. Sheikh, and G. Yu. M-degrees of quadrangle-free planar graphs. J. Graph Theory, 60(1):80-85, 2009. doi:10.1002/jgt.20346.

[12] O. V. Borodin, A. O. Ivanova, M. Montassier, and A. Raspaud. $(k, j)$ coloring of sparse graphs. Discrete Appl. Math., 159(17):1947-1953, 2011. doi:10.1016/j.dam.2011.06.021.

[13] O. V. Borodin, A. O. Ivanova, M. Montassier, and A. Raspaud. (k,1)coloring of sparse graphs. Discrete Math., 312(6):1128-1135, 2012. doi: 10.1016/j.disc.2011.11.031.

[14] O. V. Borodin, A. Kostochka, and M. Yancey. On 1-improper 2coloring of sparse graphs. Discrete Math., 313(22):2638-2649, 2013. doi:10.1016/j.disc.2013.07.014.

[15] P. Bose, M. Smid, and D. R. Wood. Light edges in degree-constrained graphs. Discrete Math., 282(1-3):35-41, 2004. doi:10.1016/j.disc.2003.12.003.

[16] M. Chen and A. Raspaud. On $(3,1)^{*}$-choosability of planar graphs without adjacent short cycles. Discrete Applied Mathematics, 162(C):159-166, 2014. doi:10.1016/j.dam.2013.09.009.

[17] M. Chen, A. Raspaud, and W. Wang. A $(3,1)^{*}$-choosable theorem on planar graphs. Journal of Combinatorial Optimization, 32(3):927-940, 2016. doi: $10.1007 / \mathrm{s} 10878-015-9913-7$.

[18] I. Choi and L. Esperet. Improper coloring of graphs on surfaces. arXiv: 1603.02841, 2016.

[19] I. Choi and A. Raspaud. Planar graphs with girth at least 5 are $(3,5)$-colorable. Discrete Math., 338(4):661-667, 2015. doi:10.1016/j.disc.2014.11.012.

[20] Y. Colin de Verdière. Sur un nouvel invariant des graphes et un critère de planarité. J. Combin. Theory Ser. B, 50(1):11-21, 1990.

[21] Y. Colin de Verdière. On a new graph invariant and a criterion for planarity. In Graph structure theory, volume 147 of Contemp. Math., pages 137-147. Amer. Math. Soc., 1993. doi: 10.1090/conm/147/01168.

[22] J. H. Conway and C. M. Gordon. Knots and links in spatial graphs. J. Graph Theory, 7:445-453, 1983. doi:10.1002/jgt.3190070410.

[23] R. Corrêa, F. Havet, and J.-S. Sereni. About a Brooks-type theorem for improper colouring. Australas. J. Combin., 43:219-230, 2009. https://ajc. maths.uq.edu.au/pdf/43/ajc_v43_p219.pdf.

[24] L. Cowen, W. Goddard, and C. E. Jesurum. Defective coloring revisited. J. Graph Theory, 24(3):205-219, 1997. doi : 10.1002/(SICI) 1097-0118(199703) 24:3<205: :AID-JGT2>3.0.C0;2-T.

[25] L. J. Cowen, R. H. Cowen, and D. R. Woodall. Defective colorings of graphs in surfaces: partitions into subgraphs of bounded valency. J. Graph Theory, 10 
(2):187-195, 1986. doi:10.1002/jgt.3190100207.

[26] E. de Klerk, D. V. Pasechnik, and G. Salazar. Book drawings of complete bipartite graphs. Discrete Appl. Math., 167:80-93, 2014. doi: $10.1016 / \mathrm{j}$. dam.2013.11.001.

[27] R. Diestel. Graph theory, volume 173 of Graduate Texts in Mathematics. Springer, 4th edition, 2010.

[28] P. Dorbec, T. Kaiser, M. Montassier, and A. Raspaud. Limits of near-coloring of sparse graphs. J. Graph Theory, 75(2):191-202, 2014. doi:10.1002/jgt.21731.

[29] V. Dujmović and D. R. Wood. On linear layouts of graphs. Discrete Math. Theor. Comput. Sci., 6(2):339-358, 2004. http://dmtcs.episciences.org/ 317.

[30] N. Eaton and T. Hull. Defective list colorings of planar graphs. Bull. Inst. Combin. Appl, 25:79-87, 1999.

[31] K. Edwards, D. Y. Kang, J. Kim, S. Oum, and P. Seymour. A relative of Hadwiger's conjecture. SIAM J. Discrete Math., 29(4):2385-2388, 2015. doi: $10.1137 / 141002177$.

[32] J. Foisy. Intrinsically knotted graphs. J. Graph Theory, 39(3):178-187, 2002. doi: $10.1002 /$ jgt.10017.

[33] Z. Füredi and J. Kahn. On the dimensions of ordered sets of bounded degree. Order, 3(1):15-20, 1986. doi: 10.1007/BF00403406.

[34] E. Gethner and T. Sulanke. Thickness-two graphs. II. More new ninecritical graphs, independence ratio, cloned planar graphs, and singly and doubly outerplanar graphs. Graphs Combin., 25(2):197-217, 2009. doi: $10.1007 / \mathrm{s} 00373-008-0833-5$.

[35] N. Goldberg, T. W. Mattman, and R. Naimi. Many, many more intrinsically knotted graphs. Algebr. Geom. Topol., 14(3):1801-1823, 2014. doi: 10.2140/agt.2014.14.1801.

[36] D. J. Harvey and D. R. Wood. Average degree conditions forcing a minor. Electron. J. Combin., 23(1):\#P1.42, 2016. http://www.combinatorics.org/ ojs/index.php/eljc/article/view/v23i1p42/.

[37] F. Havet and J.-S. Sereni. Improper choosability of graphs and maximum average degree. J. Graph Theory, 52(3):181-199, 2006. doi:10.1002/jgt.20155.

[38] W. He, X. Hou, K.-W. Lih, J. Shao, W. Wang, and X. Zhu. Edge-partitions of planar graphs and their game coloring numbers. J. Graph Theory, 41(4): 307-317, 2002. doi: 10.1002/jgt.10069.

[39] L. S. Heath and S. V. Pemmaraju. Stack and queue layouts of posets. SIAM J. Discrete Math., 10(4):599-625, 1997. doi:10.1137/S0895480193252380.

[40] L. S. Heath and A. L. Rosenberg. Laying out graphs using queues. SIAM J. Comput., 21(5):927-958, 1992. doi:10.1137/0221055.

[41] K. Hendrey and D. R. Wood. The extremal function for Petersen minors. arXiv: 1508.04541, 2016. 
[42] J. P. Hutchinson. Coloring ordinary maps, maps of empires and maps of the moon. Math. Mag., 66(4):211-226, 1993. doi:10.2307/2690733.

[43] J. Ivančo. The weight of a graph. Ann. Discrete Math., 51:113-116, 1992.

[44] B. Jackson and G. Ringel. Variations on Ringel's earth-moon problem. Discrete Math., 211(1-3):233-242, 2000. doi: 10.1016/S0012-365X (99)00278-2.

[45] I. T. Jakobsen. Weakenings of the conjecture of Hadwiger for 8- and 9-chromatic graphs. Technical Report 22, Matematisk Institut, Aarhus Universitet, Denmark, 1971.

[46] S. Jendrol' and T. Madaras. On light subgraphs in plane graphs of minimum degree five. Discuss. Math. Graph Theory, 16(2):207-217, 1996.

[47] S. Jendrol' and M. Tuhársky. A Kotzig type theorem for non-orientable surfaces. Mathematica Slovaca, 56(3):245-253, 2006. http://dml.cz/dmlcz/130967.

[48] S. Jendrol' and H.-J. Voss. Light subgraphs of multigraphs on compact 2dimensional manifolds. Discrete Math., 233(1-3):329-351, 2001.

[49] S. Jendrol' and H.-J. Voss. Light subgraphs of graphs embedded in 2-dimensional manifolds of Euler characteristic $\leqslant 0$. A survey. In Paul Erdős and his Mathematics, II, volume 11 of Bolyai Soc. Math. Stud., pages 375-411. János Bolyai Math. Soc., 2002.

[50] C. D. Keys. Graphs critical for maximal bookthickness. Pi Mu Epsilon J., 6: 79-84, 1975.

[51] J. Kim, A. Kostochka, and X. Zhu. Improper coloring of sparse graphs with a given girth, II: constructions. J. Graph Theory, 81(4):403-413, 2016. doi: 10.1002/jgt.21886.

[52] J. Komlós and E. Szemerédi. Topological cliques in graphs. II. Combin. Probab. Comput., 5(1):79-90, 1996.

[53] A. V. Kostochka. The minimum Hadwiger number for graphs with a given mean degree of vertices. Metody Diskret. Analiz., 38:37-58, 1982.

[54] A. V. Kostochka. Lower bound of the Hadwiger number of graphs by their average degree. Combinatorica, 4(4):307-316, 1984. doi : 10.1007/BF02579141.

[55] A. V. Kostochka and N. Prince. On $K_{s, t}$-minors in graphs with given average degree. Discrete Math., 308(19):4435-4445, 2008. doi: $10.1016 /$ j.disc. 2007.08 .041

[56] A. V. Kostochka and N. Prince. Dense graphs have $K_{3, t}$ minors. Discrete Math., 310(20):2637-2654, 2010. doi: 10.1016/j.disc.2010.03.026.

[57] A. V. Kostochka and N. Prince. On $K_{s, t}$-minors in graphs with given average degree, II. Discrete Math., 312(24):3517-3522, 2012. doi: $10.1016 /$ j.disc.2012.08.004

[58] D. Kühn and D. Osthus. Complete minors in $K_{s, s}$ free graphs. Combinatorica, 25(1):49-64, 2005. doi: 10.1007/s00493-005-0004-8.

[59] D. Kühn and D. Osthus. Forcing unbalanced complete bipartite minors. European J. Combin., 26(1):75-81, 2005. doi:10.1016/j.ejc.2004.02.002. 
[60] K.-W. Lih, Z. Song, W. Wang, and K. Zhang. A note on list improper coloring planar graphs. Appl. Math. Lett., 14(3):269-273, 2001. doi : 10.1016/S0893-9659(00)00147-6.

[61] W. Mader. Homomorphiesätze für Graphen. Math. Ann., 178:154-168, 1968. doi: 10.1007/BF01350657.

[62] P. Mutzel, T. Odenthal, and M. Scharbrodt. The thickness of graphs: a survey. Graphs Combin., 14(1):59-73, 1998. doi:10.1007/PL00007219.

[63] J. Nešetřil and P. Ossona de Mendez. First order properties on nowhere dense structures. J. Symb. Log., 75(3):868-887, 2010. doi: $10.2178 / \mathrm{jsl} / 1278682204$.

[64] J. Nešetřil and P. Ossona de Mendez. On nowhere dense graphs. European Journal of Combinatorics, 32(4):600-617, 2011. doi:10.1016/j .ejc.2011.01.006.

[65] J. Nešetřil and P. Ossona de Mendez. Sparsity (Graphs, Structures, and Algorithms), volume 28 of Algorithms and Combinatorics. Springer, 2012.

[66] J. Nešetřil, P. Ossona de Mendez, and D. R. Wood. Characterisations and examples of graph classes with bounded expansion. European J. Combinatorics, 33(3):350-373, 2011. doi:10.1016/j.ejc.2011.09.008.

[67] M. Ozawa and Y. Tsutsumi. Primitive spatial graphs and graph minors. Rev. Mat. Complut., 20(2):391-406, 2007. doi:10.5209/rev_REMA.2007.v20.n2.16496.

[68] J. Pach and G. Tóth. Graphs drawn with few crossings per edge. Combinatorica, 17(3):427-439, 1997. doi:10.1007/BF01215922.

[69] S. V. Pemmaraju. Exploring the Powers of Stacks and Queues via Graph Layouts. PhD thesis, Virginia Polytechnic Institute and State University, U.S.A., 1992.

[70] J. L. Ramírez Alfonsín. Knots and links in spatial graphs: a survey. Discrete Math., 302(1-3):225-242, 2005. doi:10.1016/j.disc.2004.07.035.

[71] B. Reed and D. R. Wood. Forcing a sparse minor. Combin. Probab. Comput., 25:300-322, 2016. doi:10.1017/S0963548315000073.

[72] G. Ringel. Färbungsprobleme auf Flächen und Graphen, volume 2 of Mathematische Monographien. VEB Deutscher Verlag der Wissenschaften, Berlin, 1959.

[73] G. Ringel. Das Geschlecht des vollständigen paaren Graphen. Abh. Math. Sem. Univ. Hamburg, 28:139-150, 1965.

[74] N. Robertson, P. D. Seymour, and R. Thomas. Hadwiger's conjecture for $K_{6^{-}}$ free graphs. Combinatorica, 13(3):279-361, 1993. doi:10.1007/BF01202354.

[75] N. Robertson, P. D. Seymour, and R. Thomas. A survey of linkless embeddings. In N. Robertson and P. D. Seymour, editors, Graph structure theory. Proc. of AMS-IMS-SIAM Joint Summer Research Conf. on Graph Minors, volume 147 of Contempory Mathematics, pages 125-136. American Mathematical Society, 1993. 
[76] N. Robertson, P. Seymour, and R. Thomas. Petersen family minors. J. Combin. Theory Ser. B, 64(2):155-184, 1995. doi:10.1006/jctb.1995.1031.

[77] H. Sachs. On a spatial analogue of Kuratowski's theorem on planar graphs an open problem. In M. Borowiecki, J. W. Kennedy, and M. M. Syslo, editors, Proc. Conf. on Graph Theory, volume 1018 of Lecture Notes in Mathematics, pages 230-241. Springer, 1983.

[78] A. Schrijver. Minor-monotone graph invariants. In Surveys in combinatorics, volume 241 of London Math. Soc. Lecture Note Ser., pages 163-196. Cambridge Univ. Press, 1997. doi: 10.1017/CB09780511662119.007.

[79] F. Shahrokhi, L. A. Székely, O. Sýkora, and I. Vrťo. Drawings of graphs on surfaces with few crossings. Algorithmica, 16(1):118-131, 1996. doi: 10.1007/BF02086611.

[80] R. Škrekovski. List improper colorings of planar graphs with prescribed girth. Discrete Math., 214(1-3):221-233, 2000. doi: 10.1016/S0012-365X (99)00145-4.

[81] R. Thomas and P. Wollan. An improved linear edge bound for graph linkages. European J. Combin., 26(3-4):309-324, 2005. doi: $10.1016 / j \cdot e j c .2004 .02 .013$.

[82] A. Thomason. An extremal function for contractions of graphs. Math. Proc. Cambridge Philos. Soc., 95(2):261-265, 1984. doi: 10.1017/S0305004100061521.

[83] A. Thomason. The extremal function for complete minors. J. Combin. Theory Ser. B, 81(2):318-338, 2001. doi:10.1006/jctb.2000.2013.

[84] H. van der Holst. On the graph parameters of Colin de Verdière. In Ten years LNMB, pages 37-44. Math. Centrum Centrum Wisk. Inform., Amsterdam, 1997.

[85] H. van der Holst, L. Lovász, and A. Schrijver. The Colin de Verdière graph parameter. In Graph theory and Combinatorial Biology, volume 7 of Bolyai Soc. Math. Stud., pages 29-85. János Bolyai Math. Soc., 1999.

[86] Y. Wang and L. Xu. Improper choosability of planar graphs without 4-cycles. SIAM J. Discrete Math., 27(4):2029-2037, 2013. doi:10.1137/120885140.

[87] D. R. Wood. Cliques in graphs excluding a complete graph minor. Electron. J. Combin., 23(3):\#P3.18, 2016. http://www.combinatorics.org/ojs/index. php/eljc/article/view/v23i3p18/.

[88] R. G. Wood and D. R. Woodall. Defective choosability of graphs without small minors. Electron. J. Combin., 16(1):\#R92, 2009. http://www.combinatorics . org/Volume_16/Abstracts/v16i1r92.html.

[89] D. R. Woodall. Defective choosability of graphs in surfaces. Discuss. Math. Graph Theory, 31(3):441-459, 2011. doi: 10.7151/dmgt.1557.

[90] M. Yancey. Thickness for improper colorings, 2012. http://www.math. illinois.edu/ dwest/regs/impthic.html. 
[91] H. Zhang. On $(4,1)^{*}$-choosability of toroidal graphs without chordal 7-cycles and adjacent 4-cycles. Commentationes Mathematicae Universitatis Carolinae, 54(3):339-344, 2013. http://hdl.handle.net/10338.dmlcz/143305.

[92] H. Zhang. $(3,1)^{*}$-choosability of graphs of nonnegative characteristic without intersecting short cycles. Proceedings - Mathematical Sciences, 126(2):159-165, 2016. doi: 10.1007/s12044-016-0272-9.

[93] L. Zhang. A $(3,1)^{*}$-choosable theorem on toroidal graphs. Discrete Applied Mathematics, 160(3):332-338, 2012. doi:10.1016/j.dam.2011.10.019.

Patrice Ossona de Mendez

Centre d'Analyse et de Mathématiques Sociales (CNRS, UMR 8557)

190-198 avenue de France, 75013 Paris, France

- AND -

Computer Science Institute of Charles University (IUUK)

Malostranské nám.25, 11800 Praha 1, Czech Republic

E-mail address: pom@ehess.fr

SANG-IL OUm

Department of Mathematical Sciences, Kaist

Daejeon, South Korea

E-mail address: sangil@kaist.edu

DAVID R. WOOD

School of Mathematical Sciences, Monash University

Melbourne, Australia

E-mail address: david.wood@monash.edu 